\title{
THE EQUILIBRIA AND EVOLUTIONS OF MAGNETIZED, ROTATING, ISOTHERMAL CLOUDS. IV. QUASI-STATIC EVOLUTION
}

\author{
KOHJI TOMISAKA \\ Faculty of Education, Niigata University \\ SATORU IKEUCHI \\ National Astronomical Observatory \\ AND \\ TAKASHI NAKAMURA \\ Research Institute for Fundamental Physics \\ Received 1989 June 16; accepted 1990 April 11
}

\begin{abstract}
The evolution of a magnetized, rotating, isothermal cloud is studied assuming quasi-static evolution. The evolution is driven by the plasma drift (ambipolar diffusion) and magnetic braking. Mass and angular momentum are transferred inwardly across the magnetic flux tube due to the plasma drift. Since the rotating cloud winds the magnetic field, torsional Alfvén waves transport angular momentum away from the cloud. We consider the cloud rotating with an angular momentum parallel to the magnetic field, which is immersed in a static external pressure and is threaded by a uniform magnetic field at infinity. The problem is characterized by four parameters which determine the initial equilibrium structure (cloud mass, magnetic flux, ratio of thermal pressure to magnetic pressure, and total angular momentum) and a parameter which expresses the efficiency of magnetic braking, i.e., density of the ambient medium $\rho_{a}$. Due to the plasma drift, the mass-tomagnetic flux ratio at the center increases and, accordingly, the central density $\rho_{c}$ increases. After the epoch when the mass-to-flux ratio exceeds a critical value, there exist no equilibrium solutions and the cloud begins to collapse dynamically. This occurs in $\tau_{P} \simeq 16\left(G \rho_{c}\right)^{1 / 2} \sim$ several Myr for molecular cores with density $2 \times 10^{4}$ $\mathrm{cm}^{-3}$. The angular momentum is lost by magnetic braking in a time $\tau_{\|} \simeq \sigma /\left(2 \rho_{a} V_{\mathrm{A}}\right) \sim$ several Myr, where $\sigma$ and $V_{\mathrm{A}}$ denote, respectively, column density of the cloud and Alfvén speed in the ambient medium, in agreement with the analytical result by Mouschovias and Paleologou. Depending on the relative magnitude of $\tau_{P}$ and $\tau_{\|}$, the final angular momentum kept in the cloud varies. In the final phase of the quasi-static evolution, as long as the magnetic flux decreases by $\sim \frac{1}{2}$ to the critical value, the cloud central density increases by a factor of more than 100 .

Subject headings: hydromagnetics - interstellar: magnetic fields - nebulae: general nebulae: internal motions
\end{abstract}

\section{INTRODUCTION}

Dense parts of the molecular cloud, in which the star formation process seems to proceed, are often found with elongated shape (e.g., Taurus molecular cloud; Heyer et al. 1987). This suggests that the cloud collapsed preferentially along the direction parallel to the global magnetic field and/or parallel to the cloud's initial angular momentum. Actually the magnetic field strength in the cloud has been measured recently with Zeeman splitting (Crutcher et al. 1988; Goodman et al. 1989). The authors indicate the magnetic field of 10-30 $\mu \mathrm{G}$ exists in the cloud. Further, the observation of polarization in the near-IR from background stars shows that the magnetic field runs perpendicularly to the major axis of the cloud (Tamura et al. 1987). As for the angular momentum, the rotation rate of 0.2-6 $\mathrm{km} \mathrm{s}^{-1} \mathrm{pc}^{-1}$ is reported (Goldsmith and Arquilla 1985) in 16 dark cloud regions, and the directions of angular momentum are often found perpendicular to the major axes of clouds (Heyer et al. 1987). If the cloud were to collapse from the diffuse cloud with density $n \sim 1 \mathrm{~cm}^{-3}$, strictly conserving the angular momentum which was shared from the galactic rotation, the rotation rate of the cloud would be $\Omega_{J=\text { const }}$ $\sim 3\left(n / 1000 \mathrm{~cm}^{-3}\right)^{2 / 3} \mathrm{~km} \mathrm{~s}^{-1} \mathrm{pc}^{-1}$ (Mouschovias 1987). There is a possibility that the effect of rotation becomes important in molecular clouds. In the present paper, we will study the evolution of the rotating magnetized cloud.

The evolution of the cloud can be sketched to a certain extent by studying the hydrostatic equilibrium solutions of the cloud. There exists a maximum mass which can be supported against the self-gravity by magnetic field, thermal pressure, and rotation. We will call it the "critical mass" $M_{\mathrm{cr}}$. The cloud with mass greater than the critical mass, i.e., $M_{\mathrm{cl}}>M_{\mathrm{cr}}$, has no hydrostatic equilibrium solution and collapses dynamically (supercritical cloud); on the other hand, the cloud with $M_{\mathrm{cl}}<$ $M_{\text {cr }}$ will settle into an equilibrium state (subcritical cloud) (e.g., Mouschovias and Spitzer 1976). Here we summarize how the critical mass is related to the external pressure, the magnetic flux threading the cloud, and the specific angular momentum of the cloud. As for the isothermal spherical cloud with no magnetic field nor angular momentum, the critical mass is expressed as (Bonner 1956; Ebert 1955)

$$
M_{\mathrm{BE}}=52.6 \frac{c_{s}^{4}}{(4 \pi G)^{3 / 2} p_{\mathrm{ext}}^{1 / 2}},
$$

where $c_{s}, p_{\text {ext }}$, and $G$ represent, respectively, the isothermal sound speed, the external pressure, and the gravitational constant. For a magnetized but nonrotating cloud, Mouschovias 
and Spitzer (1976; see also Spitzer 1978, p. 239) first obtained the expression of the critical mass using the numerical results of Mouschovias (1976b) as

$$
M_{\mathrm{mag}} \simeq 62\left[1-\left(\frac{0.126}{G^{1 / 2} M_{\mathrm{mag}} / \Phi_{B}}\right)^{2}\right]^{-3 / 2} \frac{c_{s}^{4}}{(4 \pi G)^{3 / 2} p_{\mathrm{ext}}^{1 / 2}},
$$

which is related to the mass-flux ratio of the cloud $M_{\text {mag }} / \Phi_{B}$. Further, related to the distribution of mass against the magnetic flux in the cloud, Mouschovias (1978) pointed out that the magnetic flux threading the cloud core plays more important role to support the cloud than that threading the outer layer. Recently Tomisaka, Ikeuchi, and Nakamura (1988b; hereafter Paper II) has exactly confirmed this point by comparing the magnetohydrostatic solutions with different mass distributions against magnetic flux other than Mouschovias $(1976 b)$. Finally, fitting by the same functional form as equation (1.2), the critical mass $M_{\text {mag }}$ is adequately written in terms of the mass-flux ratio at the center of the cloud $\left|d M / d \Phi_{B}\right|_{c}$ as

$$
M_{\mathrm{mag}} \simeq 62\left[1-\left(\frac{0.17}{G^{1 / 2}\left|d M / d \Phi_{B}\right|_{c}}\right)^{2}\right]^{-3 / 2} \frac{c_{s}^{4}}{(4 \pi G)^{3 / 2} p_{\mathrm{ext}}^{1 / 2}},
$$

which becomes identical with equation (1.2) when we take the same mass distribution against flux as that of the spherical cloud threaded by the uniform magnetic field. Finally, in the most general case of the magnetized cloud with a specific angular momentum $j$ parallel to the global magnetic field, the critical mass is approximated by

$$
M_{\mathrm{cr}} \simeq\left[M_{\mathrm{mag}}^{2}+\left(\frac{4.8 c_{s} j}{G}\right)^{2}\right]^{1 / 2},
$$

using $M_{\text {mag }}$ of equation (1.2a) (Tomisaka, Ikeuchi, and Nakamura 1989; hereafter Paper III). Using a relation between the column density and the central density in gravitating thin disks (eq. [3.6] of Paper II), the condition that $M_{\mathrm{cl}}=M_{\text {rot }} \equiv$ $4.8 c_{s} j / G$ gives an almost similar condition that the uniform rotating disk is marginally stable for fragmentation (Spitzer 1978, p. 291).

Here we study the evolution of subcritical clouds $\left(M_{\mathrm{cl}}<\right.$ $M_{\text {cr }}$ ). Processes driving the evolution of subcritical clouds are plasma drift and magnetic braking:

1. Plasma drift.-In the weakly ionized cloud, ions are coupled with the magnetic field, but neutral atoms and molecules are coupled with it only indirectly, i.e., through twobody collisions between ions and neutral particles. Thus, the neutral particles drift across the magnetic flux. Since the mass and simultaneously angular momentum in a flux tube are transferred by this plasma drift, the distribution of mass and angular momentum changes. The cloud evolves so as to follow the new distribution of mass and angular momentum.

2. Magnetic braking.-The rotating cloud twists the magnetic field and drives torsional Alfvén wave propagating along the magnetic field. The torsional Alfven wave transfers the angular momentum of the cloud away. Thus, the cloud spins down. Magnetic braking changes the angular momentum itself.

Evolution of nonrotating isothermal clouds has been studied by several authors. Shu (1983), Scott (1984), Paleologou and Mouschovias (1983), and Mouschovias, Paleologou, and Fiedler (1985) studied the plane-parallel cloud extending infinitely with the magnetic field parallel to the slab. Nakano $(1979,1982)$ studied the evolution of a realistic axisymmetric cloud threaded by magnetic field parallel to the symmetric axis. He showed that as the mass-to-flux ratio at the center increases due to the plasma drift, the central density increases (see also Lizano and Shu 1989). With the increase of the density, plasma drift is accelerated, since the ionization rate decreases. When the central density reaches $10^{9-10} \mathrm{~cm}^{-3}$, at that time the cloud almost reaches the critical state $\left(M_{\mathrm{cl}} \simeq M_{\mathrm{cr}}\right)$, the mass-to-flux ratio at the center increases only $1.3-2$ times that of the initial state, while the central density increases by a factor of 100 (Nakano 1982). From equation (1.2a), the increase of the central mass-to-flux ratio reduces the critical mass. After the critical mass becomes smaller than the cloud mass itself, there is no equilibrium configuration (Paper II) and the central part of the cloud begins to collapse dynamically. Therefore, the central part of a cloud cannot lose more than $50 \%$ of its initial magnetic flux before the central density reaches $\sim 10^{9-10}$ $\mathrm{cm}^{-3}$, when the initial state is not so far from the critical state. (Contrarily, the plane-parallel cloud can increase its mass-toflux ratio, $\rho / B$, by several orders of magnitude, when the density $\sim 10^{8} \mathrm{~cm}^{-3}$. We will discuss this difference in $\S \mathrm{IV}$.)

In the case that angular momentum is parallel to the magnetic field, the time scale in which the angular momentum is transferred away by magnetic braking has been studied by Mouschovias and Paleologou (1980). When the disk-shaped cloud with half-height $Z$ and uniform density $\rho_{\mathrm{cl}}$ is immersed in the ambient medium with the density $\rho_{a}$ and magnetic field $B_{0}$, the time scale becomes

$$
\tau_{\|} \simeq \frac{\rho_{\mathrm{cl}} Z}{\rho_{a} V_{\mathrm{A}}},
$$

where $V_{\mathrm{A}} \equiv B_{0} /\left(4 \pi \rho_{a}\right)^{1 / 2}$ represents the Alfven speed in the ambient medium. This just corresponds to the time scale at which the moment of inertia swept by torsional Alfvén waves becomes comparable to that of the cloud (Mouschovias and Paleologou 1980). In the case that the angular momentum plays a role in supporting a cloud, loss of the angular momentum will cause a change of the structure of the cloud. As far as we know, this effect has never been studied. Further, in the rotating magnetized cloud, the change of mass distribution by plasma drift and decrease of the angular momentum by magnetic braking occur simultaneously. In the present paper, we will show the evolution of the cloud driven by these two processes. Since the evolution time scale driven by the plasma drift is $\sim 10$ times longer than the free-fall time scale (Nakano and Umebayashi 1980), we assume that the evolution is slower than the dynamical time scale of the cloud (we will discuss the validity of this assumption in $\S$ II), and we study the quasi-static evolution similar to the previous study on the plasma drift (e.g., Nakano 1982).

The plan of the present paper is as follows: In § II we describe the model and the numerical method. At the same time, the applicability of the quasi-static assumption is discussed. In § III, evolution of typical clouds is shown. We discuss the angular momentum loss rate and the difference between the present case and the plane-parallel cloud in § IV. Section $V$ is devoted to a summary of the paper.

\section{MODEL AND METHOD}

Tomisaka, Ikeuchi, and Nakamura (1988a; hereafter Paper I) formulated the method for determining the hydrostatic equilibrium solution of the rotating, magnetized isothermal cloud with an angular momentum parallel to the global magnetic 
field. Using this formulation, we can obtain hydrostatic equilibria of the clouds with arbitrary distributions of mass and angular momentum against magnetic flux. To study the quasistatic evolution of subcritical clouds, we must know how the distributions of mass and angular momentum change.

\section{a). Plasma Drift}

Quasi-static evolution driven by the plasma drift in the rotating cloud is formulated by generalizing the method for the nonrotating cloud by Nakano (1979) which adopted the scheme of magnetohydrostatics by Mouschovias (1976a). Neglecting the inertia term, the equation of hydrostatic balance for the neutral component becomes

$$
-\nabla p_{n}-\rho_{n} \nabla \psi+\rho_{n} \Omega^{2} r e_{r}+F=0
$$

where $p_{n}, \rho_{n}, \psi$, and $\Omega$ represent, respectively, the pressure and density of the neutral component, the gravitational potential, and angular rotation speed. $\boldsymbol{F}$ means the force exerted on the neutral component in unit volume through two-body collisions with ions. On the other hand, the hydrostatic balance of the ionic component becomes

$$
\frac{1}{4 \pi}(\nabla \times B) \times B-F=0,
$$

where the gravitational force, centrifugal force, and pressure force on the ionic component are all small compared with the magnetic force and neglected. Summing up equation (2.1) and (2.2), we obtain a hydrostatic equation for two-component fluid as

$$
-\nabla p_{n}-\rho_{n} \nabla \psi+\rho_{n} \Omega^{2} e_{r}+\frac{1}{4 \pi}(\nabla \times B) \times B=0 .
$$

Noticing total density $\rho \simeq \rho_{n}$, this equation is identical with the magnetohydrostatic equation for one-component fluid (eq. [I.2.1]; this means eq. [2.1] of Paper I). Therefore, there is no alteration on the method for obtaining equilibrium structure.

Friction force $\boldsymbol{F}$ is assumed proportional to the drift velocity of ions relative to neutral components $v_{D} \equiv v_{i}-v_{n}$ and is written as

$$
\boldsymbol{F}=\rho_{i} \rho_{n} \gamma\left(\boldsymbol{v}_{i}-\boldsymbol{v}_{n}\right),
$$

where the coefficient $\gamma$ represents the momentum exchange rate in a collision between a neutral atom and an ion. We take here $\gamma=4.28 \times 10^{13} \mathrm{~g}^{-1} \mathrm{~cm}^{-3} \mathrm{~s}^{-1}$ (Nakano 1979). From equations (2.1) and (2.4), the drift velocity of the magnetic field (and ions) relative to neutral components is expressed as

$$
v_{D}=\frac{F}{\rho_{i} \rho_{n} \gamma}=\frac{\nabla p_{n}+\rho_{n} \nabla \psi-\rho_{n} \Omega^{2} r e_{r}}{\rho_{i} \rho_{n} \gamma} .
$$

Ionization degree in the cloud is dependent upon the density and temperature of neutral components, ionization flux (stellar light, cosmic ray, etc.), and depletion fraction (Elmegreen 1979; Nakano 1979). Here we adopt a model by Nakano (1984) of temperature $T=10 \mathrm{~K}$, ionization rate $\zeta=10^{-17} \mathrm{~s}^{-1}$, depletion of carbon and oxygen $\delta_{\mathrm{CO}}=0.2$, and depletion of heavy metal $\delta_{M}=0.02$. In the range of $10^{2} \mathrm{~cm}^{-3} \lesssim n \lesssim 10^{8} \mathrm{~cm}^{-3}$, the ion density in the cloud is shown to be well-approximated as

$$
\rho_{i} \simeq C \rho_{n}^{1 / 2},
$$

where the numerical factor $C$ is equal to $4.46 \times 10^{-16} \mathrm{~g}^{1 / 2}$ $\mathrm{cm}^{-3 / 2}$. Note that the values of $C$ and the power of $\rho_{n}$ are not universal constants; e.g., for $n \gtrsim 10^{8} \mathrm{~cm}^{-3}$ the power of $\rho_{n}$ decreases gradually, and it reaches zero in the dense cloud as $n \gtrsim 10^{9.5} \mathrm{~cm}^{-3}$. Further, $C$ may vary by about a factor of 10 for clouds in the physical state different from that we adopted. We will use equation (2.6), noticing that a single power law is restricted for density $10^{2} \mathrm{~cm}^{-3} \leqslant n \lesssim 10^{8} \mathrm{~cm}^{-3}$. Using equation (2.6), the right-hand side of equation (2.5) can be evaluated after the equilibrium solutions obtained.

Time evolution of the mass contained inside a magnetic flux, $m\left(<\Phi_{B}\right)$, is obtained by integrating the mass flux flowing across the magnetic flux tube as

$$
\frac{\partial m\left(<\Phi_{B}\right)}{\partial t}=\int_{\Phi_{B}} \rho v_{D} \cdot d S
$$

where the surface integration is done along the magnetic flux tube $\left(\Phi_{B}=\right.$ constant $)$. The evolution of the angular momentum inside a flux tube, $L\left(<\Phi_{B}\right)$, is also expressed as

$$
\frac{\partial L\left(<\Phi_{B}\right)}{\partial t}=\int_{\Phi_{B}} \rho r^{2} \Omega v_{D} \cdot d S
$$

\section{b) Magnetic Braking}

Here we formulate the angular momentum loss rate due to magnetic braking. Because the wave front of torsional Alfvén wave propagates with the Alfvén speed $V_{\mathrm{A}}$, angular momentum flux flowing in the magnetic flux tube between $R$ and $R+\Delta R$ at a large distance from the cloud is estimated as (Spitzer 1978, p. 291)

$$
2 V_{\mathrm{A}} \rho_{a} R v_{\phi} 2 \pi R \Delta R=B_{0}\left(\frac{\rho_{a}}{\pi}\right)^{1 / 2} R v_{\phi} 2 \pi R \Delta R,
$$

where $\rho_{a}$ and $v_{\phi}$ represent, respectively, the ambient density and rotation speed of the ambient matter after the torsional Alfvén wave front reaches. Further, we assume that the wave accelerates the envelope matter to the angular velocity of the cloud $\Omega(\Phi)$ (Spitzer 1978). Then the angular momentum $\Delta L\left(\Phi_{B}\right)$ in $\Phi_{B}-\Phi_{B}+\Delta \Phi_{B}$ decreases according to

$$
\begin{aligned}
\frac{\partial \Delta L\left(\Phi_{B}\right)}{\partial t} & =-B_{0}\left(\frac{\rho_{a}}{\pi}\right)^{1 / 2} R v_{\phi} 2 \pi R \frac{\partial R}{\partial \Phi_{B}} \Delta \Phi_{B} \\
& =-\left(\frac{\rho_{a}}{\pi}\right)^{1 / 2} \frac{\Phi_{B}}{\pi B_{0}} \Omega \Delta \Phi_{B} .
\end{aligned}
$$

We assume that the cloud evolves in quasi-static fashion due to the change of angular momentum, and we will not study actual propagations of waves and angular momentum in the present paper.

In the cloud we assume "isorotation," i.e., $\Omega=$ const along a field line. The time scale necessary for angular momentum redistribution to establish the isorotation in a magnetic tube was estimated in equation (III.2.1) as $\tau \sim c_{s} /\left[\left(2 \pi G \rho_{c}\right)^{1 / 2} V_{\mathrm{A}}\right] \sim$ $\left(2 \pi G \rho_{c}\right)^{-1 / 2}$ at the central part of the cloud, which is shorter than characteristic evolutionary time scale. Outside of the cloud we assumed that $\left|B_{\phi}\right| \ll\left(B_{r}^{2}+B_{z}^{2}\right)^{1 / 2}$ and $j_{\phi}=0$. In reality there exists $B_{\phi}$, like Mouschovias and Paleologou (1980). So, in $\S$ II $d$ we will describe the validity of this assumption, that is, whether $B_{\phi}$ plays an important role or not.

\section{c) Numerical Method}

Using the magnetic potential $\Phi \equiv \Phi_{B} /(2 \pi)$, in terms of $q(\Phi)$ defined by equation (I.2.19), effective potential $\chi \equiv$ $\left[\psi-\left(r^{2} / 2\right) \Omega^{2}\right] / c_{s}^{2}$, and rotation angular speed $\Omega$, equation $(2.7)$ 
is rewritten as

$$
\begin{aligned}
& \frac{\partial m(<\Phi)}{\partial t}=\frac{1}{C \gamma} \int_{\Phi} \frac{1}{\rho^{1 / 2}}\left(e^{-\chi} \nabla q+\rho r^{2} \Omega \nabla \Omega\right) \cdot d S, \\
& \frac{\partial L(<\Phi)}{\partial t}=\frac{\Omega(\Phi)}{C \gamma} \int_{\Phi} \frac{r^{2}}{\rho^{1 / 2}}\left(e^{-\chi} \nabla q+\rho r^{2} \Omega \nabla \Omega\right) \cdot d S,
\end{aligned}
$$

where we used the Ferraro's “isorotation law" (Priest 1982, p. 107), i.e., $\Omega$ is only a function of $\Phi$. Using the cylindrical coordinate $(z, r)$, we can rewrite the integrant of equation (2.10) as

$$
\begin{aligned}
\frac{\partial m(<\Phi)}{\partial t}= & \frac{4 \pi}{C \gamma} \int_{0}^{z_{s}(\Phi)} \frac{r d z}{\rho^{1 / 2}} e^{-\chi} \\
& \times\left(\frac{d q}{d \Phi}+\frac{q}{c_{s}^{2}} r^{2} \Omega \frac{d \Omega}{d \Phi}\right)\left[\frac{\partial \Phi}{\partial r}-\left(\frac{\partial r}{\partial z}\right)_{\Phi} \frac{\partial \Phi}{\partial z}\right], \\
\frac{\partial L(<\Phi)}{\partial t}= & \frac{4 \pi \Omega(\Phi)}{C \gamma} \int_{0}^{z_{s}(\Phi)} \frac{r^{3} d z}{\rho^{1 / 2}} e^{-\chi} \\
& \times\left(\frac{d q}{d \Phi}+\frac{q}{c_{s}^{2}} r^{2} \Omega \frac{d \Omega}{d \Phi}\right)\left[\frac{\partial \Phi}{\partial r}-\left(\frac{\partial r}{\partial z}\right)_{\Phi} \frac{\partial \Phi}{\partial z}\right],
\end{aligned}
$$

where $z_{s}(\Phi)$ represents half-height of the cloud surface.

From the distributions at time $t, m(<\Phi, t)$ and $L(<\Phi, t)$, those at $t+\Delta t$ are obtained by

$$
\begin{aligned}
m(<\Phi, t+\Delta t)= & m(<\Phi, t)+\frac{\partial m(<\Phi)}{\partial t} \Delta t \\
L(<\Phi, t+\Delta t)= & L(<\Phi, t)+\frac{\partial L(<\Phi)}{\partial t} \Delta t \\
& +\int_{0}^{\Phi} \frac{\partial \Delta L / \Delta \Phi}{\partial t} d \Phi \Delta t
\end{aligned}
$$

The time step $\Delta t$ is chosen short enough to restrict the variation of central density less than $20 \%$ in one time step. Normalizing variables as equation (I.2.34), we rewrite equations (2.9) and (2.11) as

$$
\begin{gathered}
\frac{\partial m^{\prime}\left(<\Phi^{\prime}\right)}{\partial t^{\prime}}=\frac{4 \pi(4 \pi G)^{1 / 2}}{C \gamma} \int_{0}^{z_{s}^{\prime}} \frac{r^{\prime} d z^{\prime}}{\rho^{\prime 1 / 2}} e^{-\chi} \\
\times\left(\frac{d q^{\prime}}{d \Phi^{\prime}}+q^{\prime} r^{\prime 2} \Omega^{\prime} \frac{d \Omega^{\prime}}{d \Phi^{\prime}}\right)\left[\frac{\partial \Phi^{\prime}}{\partial r^{\prime}}-\left(\frac{\partial r^{\prime}}{\partial z^{\prime}}\right)_{\Phi^{\prime}} \frac{\partial \Phi^{\prime}}{\partial z^{\prime}}\right], \\
\frac{\partial L^{\prime}\left(<\Phi^{\prime}\right)}{\partial t^{\prime}}=\frac{4 \pi(4 \pi G)^{1 / 2} \Omega^{\prime}}{C \gamma} \int_{0}^{z_{s}^{\prime}} \frac{r^{\prime 3} d z^{\prime}}{\rho^{\prime 1 / 2}} e^{-\chi} \\
\times\left(\frac{d q^{\prime}}{d \Phi^{\prime}}+q^{\prime} r^{\prime 2} \Omega^{\prime} \frac{d \Omega^{\prime}}{d \Phi^{\prime}}\right)\left[\frac{\partial \Phi^{\prime}}{\partial r^{\prime}}-\left(\frac{\partial r^{\prime}}{\partial z^{\prime}}\right)_{\Phi^{\prime}} \frac{\partial \Phi^{\prime}}{\partial z^{\prime}}\right], \\
\frac{\partial}{\partial t^{\prime}}\left(\frac{\Delta L^{\prime}}{\Delta \Phi^{\prime}}\right)=-8 \pi\left(\frac{2 \rho_{a}^{\prime}}{\beta_{0}}\right)^{1 / 2} \Omega^{\prime} \Phi^{\prime},
\end{gathered}
$$

with $\rho_{a}^{\prime}=\rho_{a} / \rho_{s}, \beta_{0}=c_{s}^{2} \rho_{s} /\left(B_{0}^{2} / 8 \pi\right)$. Here the numerical factor in equation (2.13) is equal to $4 \pi(4 \pi G)^{1 / 2} /(C \gamma)=0.603$. Hereafter, all quantities are normalized as equation (I.2.34) and primes attached to the normalized quantities are omitted.

Evolution is fully specified by four parameters to determine the initial equilibrium state (Papers I and III) and ambient density $\rho_{a}$. We assume virtually that a uniform spherical cloud ("parent cloud") relaxes to the equilibrium state conserving the angular momentum and mass in each flux tube. In this case, the former four parameters are reduced to the mass of cloud $M_{\mathrm{cl}}$, the radius of the "parent cloud" $R_{\mathrm{cl}}$, the rotation speed of the "parent cloud" $\Omega_{\mathrm{cl}}$, and the ratio of thermal pressure to the magnetic pressure in ambient medium $\beta_{0} \equiv$ $p_{\text {ext }} /\left(B_{0}^{2} / 8 \pi\right)$.

The numerical code was checked by comparing results of nonrotating quasi-static evolution with that by Nakano (1979). Equilibrium structures of the cloud agree with each other within $15 \%$ relative error of density. The time scales at which the central density increases agree within $25 \%$ relative difference. These differences are regarded as coming from those of mesh number and mesh size.

\section{d) Applicability}

The system is thought to evolve in nonhomologous fashion, i.e., the cloud contracts from the center. Therefore, the dynamical time scale in this case is the free-fall time at the center, $\tau_{\mathrm{ff}, c}$. The assumption of "quasi-static" is valid only when the magnetic flux and angular momentum vary slower than $1 / \tau_{\mathrm{ff}, c}$. The time scale of plasma drift in an axisymmetric cloud is given using two time scales at the center of the cloud: the mean collision time of a neutral particle with ions $\tau_{\mathrm{ni}}$ and the free-fall time $\tau_{\mathrm{ff}}$ as

$$
\tau_{P} \simeq \frac{8}{1.4 \pi^{2}} \frac{\tau_{\mathrm{ff}}^{2}}{\tau_{\mathrm{ni}}}
$$

(Mouschovias 1989). In the present case, since $\tau_{\mathrm{ni}}$ is expressed as $\tau_{n i}=1 /\left(C \gamma \rho_{n}^{1 / 2}\right)$, the time scale of plasma drift is given in terms of the central density $\rho_{c}$ as

$$
\tau_{P} \simeq \frac{\gamma C}{1.5 \pi G \rho_{c}^{1 / 2}} \sim 15.7\left(G \rho_{c}\right)^{-1 / 2}
$$

which is similar to the value estimated from one-dimensional slab simulation by Shu (1983) within 10\%. Thus, it is shown that the time scale for plasma drift is much longer than the free-fall time at the center of the cloud.

The time scale in which the angular momentum is lost by the plasma drift is estimated by equation (1.4) as

$$
\begin{aligned}
\tau_{\|} & \simeq \rho_{c} \frac{c_{s}}{\left(4 \pi G \rho_{c}\right)^{1 / 2}}\left(\frac{4 \pi}{\rho_{a}}\right)^{1 / 2} B_{0}^{-1} \\
& \sim 70\left(\frac{\beta_{0}}{10^{-2}}\right)^{1 / 2}\left(\frac{\rho_{c} / \rho_{s}}{10^{2}}\right)\left(\frac{\rho_{s} / \rho_{a}}{10^{2}}\right)^{1 / 2} \tau_{\mathrm{ff}, c},
\end{aligned}
$$

which is ordinary longer than $\tau_{\mathrm{ff}, c}$.

Here we have assumed that neutral particles are frozen to the magnetic field and decelerated by magnetic braking. The angular momentum of the ionized matter, which is coupled with the magnetic field, is lost by magnetic braking. However, the magnetic braking of neutral matter is not a priori evident. Mouschovias and Paleologou (1986) showed that in molecular cloud cores the collision time scale of a neutral particle in a sea of ions $\tau_{\mathrm{ni}}$ is much shorter than $\tau_{\|}$, and that the plasma drift has only a small effect on the magnetic braking. The plasma drift in the azimuthal direction is shown to be unimportant in molecular cloud cores.

The configuration of the magnetic field outside of the cloud is assumed to be obtained by neglecting the toroidal field. This holds in the case of $B_{\phi} / B_{z} \simeq r \Omega / V_{\mathrm{A}} \ll 1$. Since the Alfven speed in the envelope is equal to

$$
V_{\mathrm{A}}=\frac{c_{s}}{\left(4 \pi \beta_{0} \rho_{a}^{\prime}\right)^{1 / 2}} \sim 14.4 c_{s}\left(\frac{\beta_{0}}{0.0385}\right)^{-1 / 2}\left(\frac{\rho_{a} / \rho_{s}}{10^{-2}}\right)^{-1 / 2},
$$


TABLE 1

TAKen Model Parameters

\begin{tabular}{|c|c|c|c|c|c|}
\hline Case & $M_{\mathrm{cl}}$ & $\beta_{0}$ & $R_{\mathrm{cl}}$ & $\boldsymbol{\Omega}_{\mathrm{cl}}$ & $\rho_{a}$ \\
\hline A0 & 150 & 0.0385 & 2.4 & 0.571 & 0 \\
\hline A2 & 150 & 0.0385 & 2.4 & 0.571 & $10^{-2}$ \\
\hline A4 & 150 & 0.0385 & 2.4 & 0.571 & $10^{-4}$ \\
\hline B0 & 200 & 0.0385 & 2.4 & 0.571 & 0 \\
\hline B2 & 200 & 0.0385 & 2.4 & 0.571 & $10^{-2}$ \\
\hline $\mathrm{CO}$ & 150 & 0.385 & 2.92 & 0.452 & 0 \\
\hline $\mathrm{C} 2$ & 150 & 0.385 & 2.92 & 0.452 & $10^{-2}$ \\
\hline $\mathrm{NA}^{\mathrm{a}}$ & 150 & 0.0385 & 2.4 & 0.571 & $10^{-2}$ \\
\hline $\mathrm{NC}^{\mathrm{a}}$ & 150 & 0.385 & 2.92 & 0.452 & $10^{-2}$ \\
\hline
\end{tabular}

${ }^{a}$ This case simulates the evolution without plasma drift.

if the rotation speed is $\lesssim$ sound speed $c_{s}$, this assumption is valid. (We take here the values taken in case $\mathrm{A} 2$ of $\S \mathrm{III} b$ as typical ones.)

\section{RESULTS}

\section{a) Evolution of Molecular Cores Driven by the Plasma Drift}

We studied nine cases as shown in Table 1. First we will see cases A0 and A2 which have parameters $M_{\mathrm{cl}}=150, \beta_{0}=$ $0.0385, \Omega_{\mathrm{cl}}=0.571, R_{\mathrm{cl}}=2.4$, and $\rho_{a}=10^{-2}$ (case A2). To clarify the effect of magnetic braking, we will compare results of case A2 (magnetic braking switch-on) and those of case A0 (switch-off: $\rho_{a}=0$ ). The critical mass for this cloud with the initial mass distribution is $\simeq 220$. These nondimensional parameters are translated to the dimensional values: temperature $T=10 \mathrm{~K}$, density at the surface of the cloud $n_{\mathrm{s}}=10^{3}$ $\mathrm{cm}^{-3}$, threading magnetic field strength $B_{0}=20 \mu \mathrm{G}$, radius of "parent cloud" $R_{\mathrm{cl}}=0.26 \mathrm{pc}$, cloud mass $M_{\mathrm{cl}}=10.7 M_{\odot}$, angular rotation speed of "parent cloud" $\Omega_{\mathrm{cl}}=1 \mathrm{~km} \mathrm{~s}^{-1}$ $\mathrm{pc}^{-1}$, and envelope density $\rho_{a}=10 \mathrm{~cm}^{-3,1}$ in which we model the molecular cores with density $n \sim 10^{4} \mathrm{~cm}^{-3}$ embedded in envelopes with pressure $p_{\text {ext }}=10^{4} \mathrm{~K} \mathrm{~cm}^{-3}$ (Goldsmith 1987). In this case, the unit of time is $\left(4 \pi G \rho_{\mathrm{s}}\right)^{-1 / 2}=5.585 \times 10^{5} \mathrm{yr}$. In the present paper, we use the molecular weight $\mu=2.3$.

First, we will see the evolution of the cloud taking only the plasma drift into account $\left(\rho_{a}=0\right)$. In Figure 1, we plot the structure, i.e., density and magnetic field distribution (upper panel) and rotation law of the cloud (lower panel). Figure $1 a$ shows the initial $(t=0)$ state, that is, the equilibrium structure of the cloud to which the rigidly rotating uniform spherical cloud, threaded by a uniform magnetic field, settled with conservation of mass and angular momentum in each flux tube. The initial central density $\rho_{c}$ is 16 , which means the density contrast between the center and the surface is 16 , and the cloud rotates almost rigidly. This is understood as follows: since the "parent cloud" collapses mainly in the $z$-direction without squeezing the magnetic field, the cloud at $t=0$ shows the same rotation curve as that of the "parent cloud." Figure $1 b$ shows the structure at the time $t=7.2$. Due to the increase of the mass-to-flux ratio at the center by the plasma drift, the central density has increased $\left(\rho_{c}=45\right)$. With the increase of the central

\footnotetext{
${ }^{1}$ In case A2 the envelope density is assumed as $\rho_{a}=10 \mathrm{~cm}^{-3}$, but it does not necessarily mean that actual envelope density in a dark cloud is as low as this. This is because since $\rho_{a}$ has the effect of an angular momentum absorber, only if the angular momentum is transferred with high efficiency $\simeq 1$ as eq. (2.14), $\rho_{a}$ coincides with an actual envelope density. Thus, it is not inconsistent if actual density outside cores is larger than $10 \mathrm{~cm}^{-3}$. Although the initial value of the rotation rate, $\Omega_{\mathrm{cl}}=1 \mathrm{~km} \mathrm{~s}^{-1} \mathrm{pc}^{-1}$, may be larger than the actual value, we dare to take this value to explore the process of angular momentum loss.
}

density, the rotation curve approaches flat rotation $\left(v_{\phi} \simeq\right.$ const) from the initial almost rigid-body rotation $\left(v_{\phi} \simeq \Omega_{\mathrm{cl}} r\right)$. This is partly because the matter with relatively large specific angular momentum is transferred inwardly, and partly because the cloud collapses radially, keeping the central density high. Figure $1 c$ shows the final state at which we can marginally obtain the equilibrium solution $(t=12.8)$. The central density reaches $\rho_{c}=2153$, which is 135 times as large as initial central density. However, the central mass-to-flux ratio, $G^{1 / 2}\left|d m / d \Phi_{B}\right|_{c}$, increases from $0.137(t=0)$ to $0.31(t=12.8)$. Only $56 \%$ of the magnetic flux near the center was lost in $t=12.8$. As shown in Paper III, when the density contrast is very high $\left(\rho_{c} \gg 1\right)$, the rotation curve approaches the flat rotation, and the radial density distribution approaches $\rho(r) \propto r^{-2}$ (Paper III and Kiguchi et al. 1987). In Figure 2, we show the evolution of mass and angular momentum differential distributions against magnetic flux by solid lines for case A0. This shows clearly that the mass and angular momentum are both transferred inwardly against magnetic flux.

The plasma drift seems to have two different effects: (1) to increase the mass-to-flux ratio at the center, which permits the collapse; (2) to transfer angular momentum inwardly, which opposes the collapse. As shown in Papers II and III, the structure of the cloud is affected essentially by the mass-to-flux ratio at the center not by the average one (Mouschovias 1978; Paper II) and essentially by the total angular momentum not by the angular momentum distribution (Paper III). Thus, the plasma drift plays a role in the evolution of a cloud through the increase of mass-to-flux ratio at the center, as originally argued by Mouschovias (1978), and as we also show here in the case of rotating cloud cores.

\section{b) Evolution in the Case with Magnetic Braking}

In the previous subsection, the evolution driven only by plasma drift was shown. Here we take the same cloud parameters as in case A0, but with finite ambient density $\rho_{a}=10^{-2}$.

Since the cloud parameters, $M_{\mathrm{cl}}, \Omega_{\mathrm{cl}}, \beta_{0}$, and $\boldsymbol{R}_{\mathrm{cl}}$, are the same as for case $\mathrm{A} 0$, the initial structure $(t=0)$ is the same as Figure $1 a$. In Figure $3 a$, we show the structure at the time $t=3.8$. The central density reaches $\rho_{c}=33$, which corresponds to the value at $t=5.5$ for case A0. This indicates that the central density rapidly increases due to the magnetic braking. As for the velocity field, since a part of the angular momentum was lost from the cloud, the rotation velocity $v_{\phi}$ becomes smaller than that of case A0. Further, $v_{\phi}$ decreases with $r$ in the outer part of the cloud $\left(r \gtrsim 0.7 R_{\mathrm{cl}}\right)$. The angular momentum distribution in this case is also shown in dashed lines in Figure 2. Comparing with the initial angular momentum distribution, we can see that in the outer part $\Phi \gtrsim 0.5 \Phi_{\mathrm{cl}}$ almost one-half of the initial angular momentum was lost. This is understood as follows: (1) The moment of inertia per unit magnetic flux and thus initial angular momentum decrease rapidly for $\Phi / \Phi_{\mathrm{cl}}>\frac{2}{3}$ (see Fig. 2). (2) The moment of inertia of ambient matter increases in proportion to $\Phi$ (eq. [2.9]). (3) The angular momentum was transferred inwardly by the plasma drift. Due to the third effect, the inner part $\left(\Phi \lesssim 0.2 \Phi_{\mathrm{c}}\right)$ of the cloud has almost the same angular momentum, although the angular momentum was lost by the magnetic braking.

Figure $3 b$ illustrates the final state at which we can marginally obtain the equilibrium solution $(t=7.7)$. The central density attains to $\rho_{c}=1.4 \times 10^{3}$, which is 85 times as large as the initial density. On the other hand, the mass-to-flux ratio at the center increases only from $0.137(t=0)$ to $0.27(t=7.7)$, 

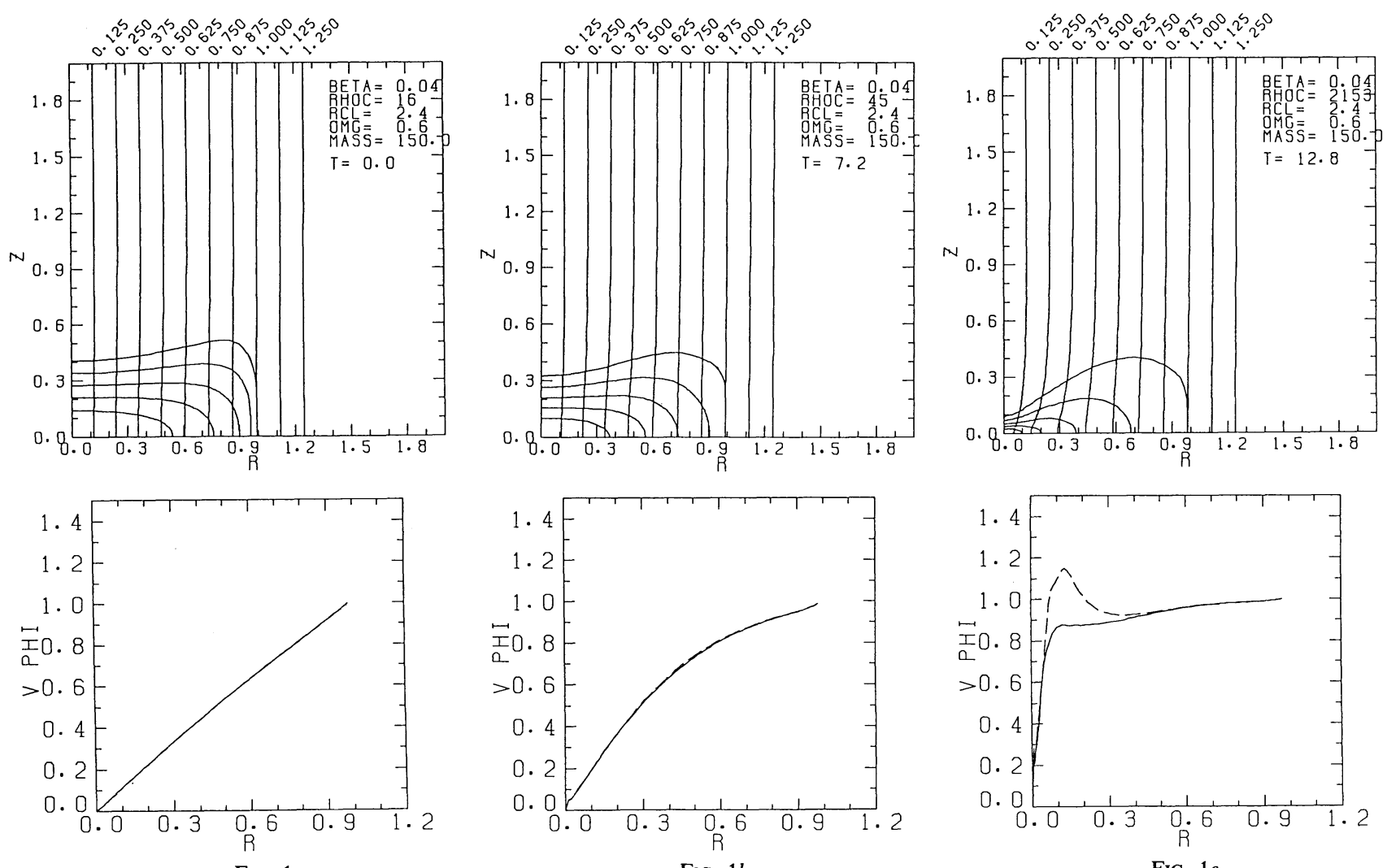

FIG. 1- The evolution of magnetized rotating clouds of case A0 (no magnetic braking) at (a) $t=0,(b) t=7.2$, and $(c) t=12.8$. In the upper panel, the magnetic

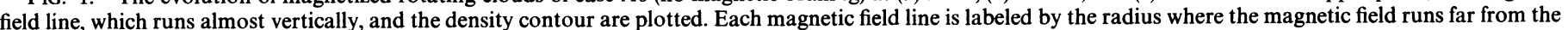

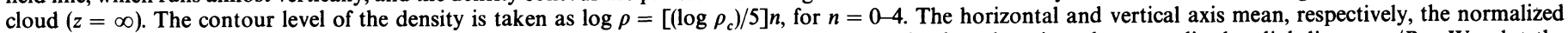

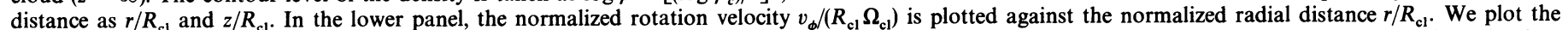

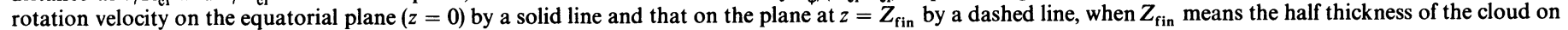
the $z$-axis. Parameters used here are summarized in Table 1.

which denotes that about one-half of the flux in the cloud core is lost in $t=7.7$. This result is very similar to that of the previous case A0. As for the structure, the density distribution resembles Figure 1c. Although the rotation is much decelerated due to the magnetic braking compared with case $\mathrm{A} 0$, the velocity structure is characterized by a rigidly rotating core plus a slowly rotating envelope, which is similar to the structure appearing in the case of $\rho_{\mathrm{c}} \gg 1$. Above $80 \%$ of the initial angular momentum of the cloud has been lost in $t=7.7$.

We show the time evolution of the central density in Figure 4. It shows that there exist two phases in evolutionary characteristics, i.e., in the first phase the density increases gradually and in the second phase density grows rapidly. The time elapses mainly in the first phase. In case A2 the cloud reaches the "critical state" faster than in case AO because the cloud loses angular momentum due to the magnetic braking during the evolution. After the equilibrium solution is no longer obtained, in other words, after the cloud mass surpasses the critical mass, the cloud will begin to collapse dynamically (Mouschovias 1976b; Nakano 1979). Time scales in which the quasi-static collapses proceed are, respectively, $12.8\left(\rho_{a}=0\right.$; case A0), $11.4\left(\rho_{a}=10^{-4}\right.$; case A4, not illustrated), and 7.7 $\left(\rho_{a}=10^{-2}\right.$; case A2). Noticing the previous normalization, we can say that in $4.3 \times 10^{6}-7.2 \times 10^{6}$ yr the central density increases by $\sim 100$ times and the mass-to-flux ratio at the center increases by $\sim 2$ times. After that, the dynamical collapse begins in the cloud core.

\section{c) Effect of Mass Difference}

To see the effect of mass differences, we will study the evolution of the cloud core with $M_{\mathrm{cl}}=200\left(14.2 M_{\odot}\right.$ in a dimensional form). The purpose of this subsection is to clarify the evolutionary difference between the core with a mass near the critical mass and that with smaller mass. The critical mass of this cloud for the initial mass distribution is equal to $\simeq 220$.

Figure 5 shows the structural change of the cloud with $M_{\mathrm{cl}}=200$ and $\rho_{a}=10^{-2}$ (case B2). The initial equilibrium structure $(t=0)$ is shown in Figure $5 a$. To support more mass than case $A$ by the same magnetic flux, the central density must be higher than that of case $A$ to keep the hydrostatic equilibrium $\left(\rho_{c}=59\right)$. The initial cloud rotates almost rigidly. At $t=1.0$ (Fig. $5 b$ ), the deceleration of rotation in the outer part $\left(r \gtrsim 0.8 R_{\mathrm{cl}}\right)$ becomes conspicuous due to the magnetic momentum loss and transfer. The mass-to-flux ratio increases from 0.183 (initial) to 0.199 . At the same time, the central density increases up to $\rho_{c}=100$ and the vertical thickness of the cloud decreases. Figure $5 c$ shows the final state $t=2.4$. Compared with the final state of case A2 (Fig. $3 b$ ), it is easily seen that the angular momentum is fairly left in the cloud. In case A2, only $20 \%$ of the initial angular momentum remains in the cloud. In contrast, the final total angular momentum, $J$, is 161 versus the initial value 263 . The maximum rotation speed remains as large as $v_{\phi} \sim 0.6 R_{\mathrm{cl}} \Omega_{\mathrm{cl}}=0.82 c_{s}$. The radial density distribution is well-approximated as $\rho(r) \propto r^{-2}$, which is char- 


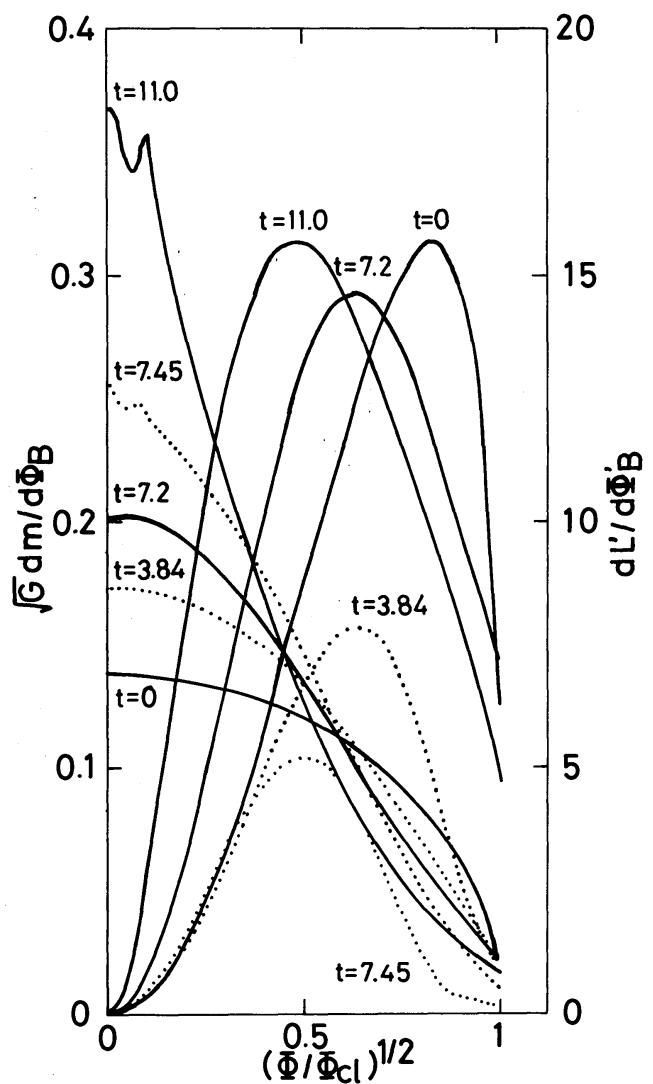

Fig. 2.-The evolution of distributions of mass and angular momentum against magnetic flux. Distributions of mass per unit magnetic flux and angular momentum per unit magnetic flux are shown for cases A0 (without magnetic braking, solid lines) and A2 (with magnetic braking, dotted lines). The lines which start from the origin show the angular momentum distributions.

acteristic of the cloud with high central density, seen in cases A0, A2, and A4. At the final state, the central density attains $\rho_{c}=4 \times 10^{3}$ and the mass-to-flux ratio is 0.25 . Also in this case, although $\rho_{c}$ increases by $\sim 70$ times that of the initial state, the mass-to-flux ratio does only by $40 \%$.

The characteristic points in case B2 are as follows: (1) The cloud/cloud core with the mass near the critical mass, i.e., the cloud with large mass-to-flux ratio, has a high central density to counterbalance the self-gravity. (2) Since the time scale in which the plasma drift changes the mass-to-flux ratio is proportional to the dynamical time scale as $\tau_{P} \simeq 15.7\left(G \rho_{c}\right)^{-1 / 2}$ (eq. [2.16]), the plasma drift works effectively in such a highdensity cloud. ${ }^{2}$ (3) The time scale in which the cloud loses its angular momentum by the magnetic braking is estimated as (Mouschovias and Paleologou 1980)

$$
\tau_{\|}=\frac{\sigma}{2 \rho_{a} V_{\mathrm{A}}},
$$

where $\sigma$ denotes the column density of the cloud threaded by a magnetic field. Comparing this case with case $A 2, \tau_{\|}$should be $4 / 3$ times as large as that of case A2 because $\sigma$ of this case is $4 / 3$ times larger. The above three points characterize the evolution of the cloud, that is, before the angular momentum is considerably lost, the mass-to-flux ratio at the center becomes larger

${ }^{2}$ This estimate is fairly correct. From the initial $\tau_{P}$ it is expected that the $e$-folding time of $G^{1 / 2}\left|d m / d \Phi_{B}\right|_{c}$ is $\tau_{P} \simeq 7$. This estimate of the time scale can explain the result that in time $t=2.4$ the mass-to-flux ratio increases by $40 \%$. than the critical value due to the plasma drift and the quasistatic evolution ends. Available time left to the cloud for quasistatic evolution is short.

Since the mass-to-flux ratio corresponds to the magnetically supported mass $M_{\text {mag }}$ (eq. [1.2a]), the final value of mass-toflux ratio depends upon a fraction at which the cloud is supported by magnetic field and thus by the rotation (eq. [2.3]). The final mass-to-flux ratios at the center for cases B0 and B2 resemble each other as $\simeq 0.25$ (case B0) and $\simeq 0.27$ (case B2). This fact indicates that the lost angular momentum $(\simeq 40 \%$ of the initial value) does not play an important role to support the cloud. Using the initial cloud parameters and equations (1.2a) and (1.3), we can estimate magnetically supported mass $M_{\text {mag }} \simeq 62\left\{1-\left[0.17 /\left(G^{1 / 2}\left|d M / d \Phi_{B}\right|_{c}\right)\right]^{2}\right\}^{-3 / 2} \simeq 1200 \quad$ and rotationally supported mass $M_{\text {rot }} \equiv 4 \pi J / M /(0.043)^{1 / 2} \simeq 80$. Therefore, in this case, the magnetically supported mass is much larger than that supported by the rotation.

\section{d) Diffuse Cloud}

As the case C, we study the cloud evolution with $M_{\mathrm{cl}}=150$, $\Omega_{\mathrm{cl}}=0.452, R_{\mathrm{cl}}=2.92$, and $\beta_{0}=0.385$. This is the weak magnetic field case, and the critical mass for this cloud is estimated as $\simeq 170$. These parameters correspond to the dimensional values as $M_{\mathrm{cl}}=34 M_{\odot}, B_{0}=3 \mu \mathrm{G}, n_{s}=10^{2} \mathrm{~cm}^{-3}, \Omega_{\mathrm{cl}}=0.25$ $\mathrm{km} \mathrm{s}^{-1} \mathrm{pc}^{-1}$, and $R_{\mathrm{cl}}=1 \mathrm{pc}$. The unit of time is $\left(4 \pi G \rho_{s}\right)^{-1 / 2}=$ $1.77 \times 10^{6} \mathrm{yr}$. These parameters correspond to the typical values of diffuse dark clouds (Goldsmith 1987). Cases C0 and $\mathrm{C} 2$ correspond, respectively, to the cases with $\rho_{a}=0$ and $\rho_{a}=$ $10^{-2}\left(1 \mathrm{~cm}^{-3}\right.$ in dimensional units). Figure $6 a$ shows the initial structure of case $\mathrm{C} 2$. The central density is not so large $\left(\rho_{c}=\right.$ $13)$, and the cloud rotates almost rigidly. The initial mass-toflux ratio at the center is 0.293 . In $t=1.7$ (Fig. $6 b$ ), the cloud rotation is decelerated, especially in the outer region $\left(\gtrsim 0.8 R_{\mathrm{cl}}\right)$. Figure $6 c$ shows the final state $(t=3.3)$. The central density attains $\rho_{c}=110$, and the mass-to-flux ratio reaches 0.34 . The rotational velocity field is divided into a rigidly rotational core, flat rotation envelope, and decelerated outer zone.

At the final state, a fair part of the angular momentum remains in the cloud (actually 150 out of the initial 231). This shows that the cloud reaches the critical state before much angular momentum is lost by the magnetic braking. As seen in Figure 4 , the time scale in which the cloud evolves quasistatically is much different between cases $\mathrm{C} 0$ and $\mathrm{C} 2$. This shows that magnetic braking plays a more important role than in the cases A and B. Comparing cases $\mathrm{C} 0$ and $\mathrm{C} 2$, the final values of the mass-to-flux ratio at the center are much different as 0.43 (case $\mathrm{C} 0$ ) and 0.34 (case $\mathrm{C} 2$ ). The cloud in case $\mathrm{C} 2$ becomes unable to support beyond $G^{1 / 2}\left|d m / d \Phi_{B}\right|_{c}>0.34$, because only $35 \%$ of the initial angular momentum was lost. This is understood from equations (1.2a) and (1.3), that is, the cloud mass which could be supported only by the rotation $M_{\text {rot }} \equiv 4 \pi J / M /(0.043)^{1 / 2} \simeq 93$ is comparable to that by magnetic field $M_{\text {mag }} \simeq 114.5$. To the evolution of the cloud with $M_{\text {mag }} \sim M_{\text {rot }}$, the decrease of angular momentum has an important role.

\section{e) Evolution Driven Purely by the Magnetic Braking}

To clarify the effect of the plasma drift to the evolution of the cloud, we will see here the evolution driven only by the magnetic braking. As in case NA2, we take $C \gamma \rightarrow \infty$ in equation (2.13) and keep other parameters the same as in case A2. Case NC2 represents the non-plasma drift version of case $\mathrm{C} 2$.

Figure $7 a$ shows the structure and rotation velocity at the 

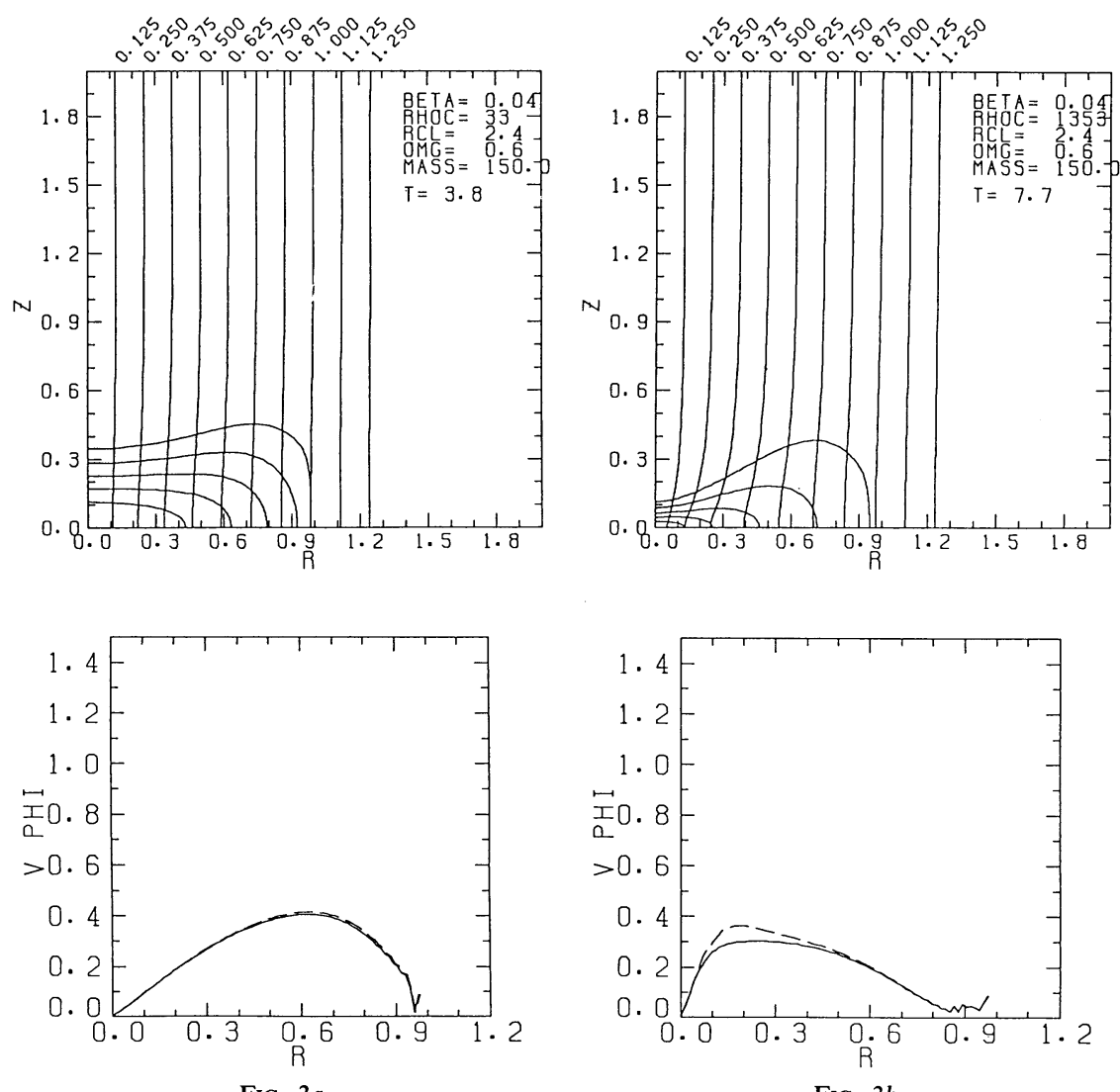

FIG. $3 a$

Fig. $3 b$

FIG. 3.-Same as Fig. 1, but for case A2 at $(a) t=3.8$ and $(b) t=7.7$. The initial state is the same as Fig. $1 a$. Due to the effect of magnetic braking, the rotational

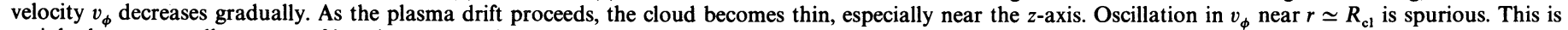

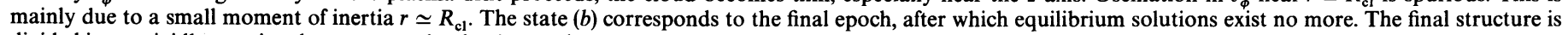
divided into a rigidly rotating dense core and a slowly rotating envelope.

time $t=10.9$ of case NA2. Although the rotation velocity is much slower than that of the initial stage (Fig. 1a, lower panel), the structure is very similar to the initial one (Fig. 1a, upper panel). The angular momentum decreases from $197(t=0)$ to $23(t=10.9)$. As shown in Figure 4, the central density keeps almost constant, i.e., within $20 \%$ relative variation. Figure $7 b$ shows the final stage of the quasi-static evolution of the case NC2 $(t=7.5$ ). Compared with the initial stage (Fig. $6 a$ ), it is

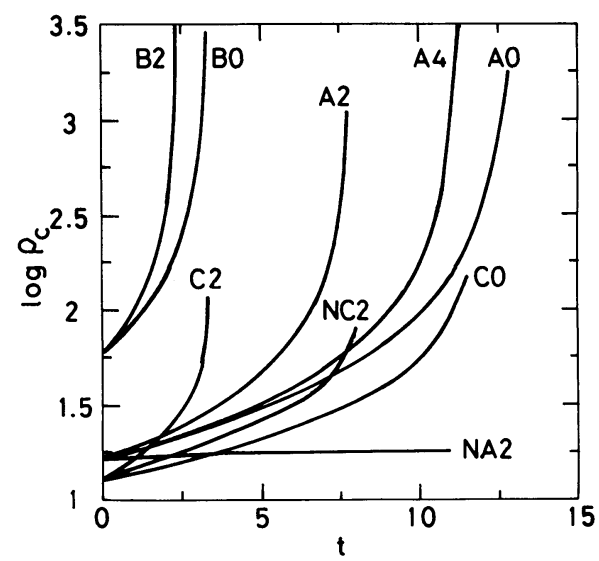

FIG. 4.-The time evolution of the central density $\rho_{c}$. It is shown that at first $\rho_{c}$ increases gradually, but finally it increases abruptly, almost vertically, in this figure. seen that the central density much increases $(13 \rightarrow 54)$ and the cloud contracts as a whole. From Figure 4, it is shown that in contrast to case NA2 this cloud evolves even without plasma drift. In this case, the structure is largely changed only by the effect of magnetic braking.

From what does the difference between cases NA2 and NC2 come? As shown in $\S$ III $c$, in case A the cloud is mainly supported magnetically $\left(M_{\text {mag }} \gg M_{\text {rot }}\right)$, whereas in case $\mathrm{C}$ the cloud rotation plays a comparable role to the magnetic field $\left(M_{\text {mag }} \sim M_{\text {rot }}\right)$. In the latter case, the decrease of angular momentum greatly affects the structure of the cloud. Thus the cloud evolves even without the plasma drift. Contrarily, in the former case $\left(M_{\text {mag }} \gg M_{\text {rot }}\right)$ the magnetic braking lets the cloud rotate slowly but does not change the structure of the cloud.

\section{f) Density-Magnetic Field Relation}

Comparing a sequence of solutions with the same magnetic flux, it is shown that the magnetic field at the center $B_{c}$ increases in proportion to $\rho_{c}^{1 / 2}$ for $\rho_{c} \gtrsim \beta_{0}^{-1}$ (§ III $e$ of Paper II; Mouschovias 1976b). This corresponds to the fact that the density increases with keeping $\rho_{c} \propto \sigma^{2} \propto B_{c}^{2}$ (see Paper II). Here we will see how the cloud evolves quasi-statically in the $\left(\rho_{c}-B_{c}\right)$-plane.

Figure 8 shows the evolutionary tracks in the $\left(\rho_{c}-B_{c}\right)$ plane. For $\rho_{c} \gtrsim 300$ (in the case of $\beta_{0}=0.0385$ ) and $\rho_{c} \gtrsim 10$ (in the case of $\left.\beta_{0}=0.385\right), B_{c}$ correlates well with the power of $\rho_{c}$, as $B_{c} \propto \rho_{c}^{\alpha}$. The power index $\alpha$ is estimated as $\alpha \simeq 0.43-0.44$ for 

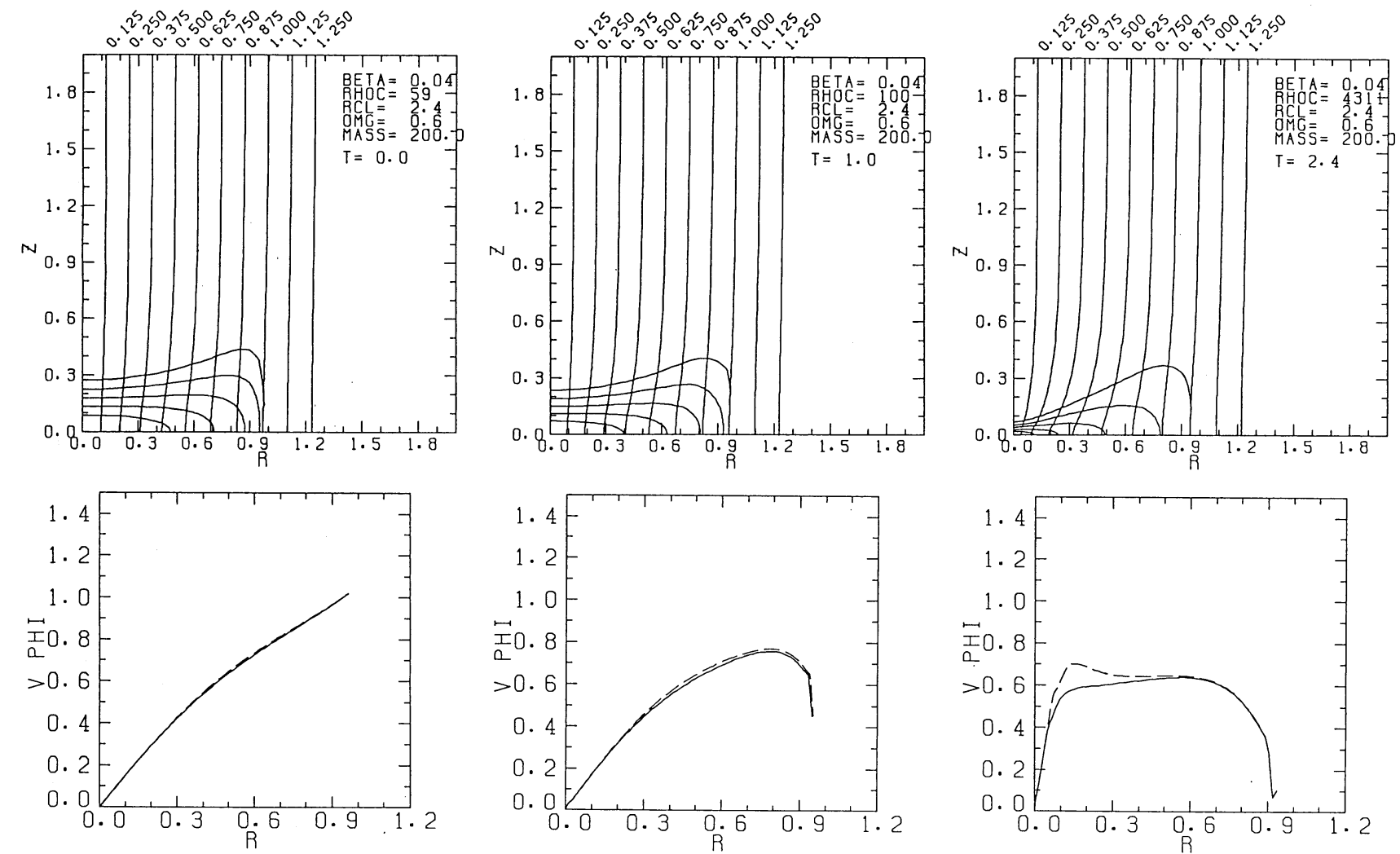

Fig. $5 a$

FIG. $5 b$

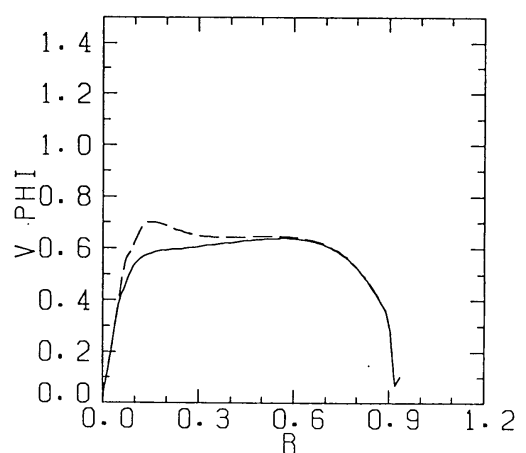

FIG. $5 c$

Fig. 5.-Same as Fig. 1, but for case B2 at $(a) t=0,(b) t=1.0$ and $(c) t=2.4$. Because this cloud has a larger mass than case A, the plasma drift works efficiently. Therefore, the cloud evolves much faster than case A2. From the figure of $v_{\phi}$, it is shown that even in the final phase (c) the cloud contains a fairly large angular momentum.
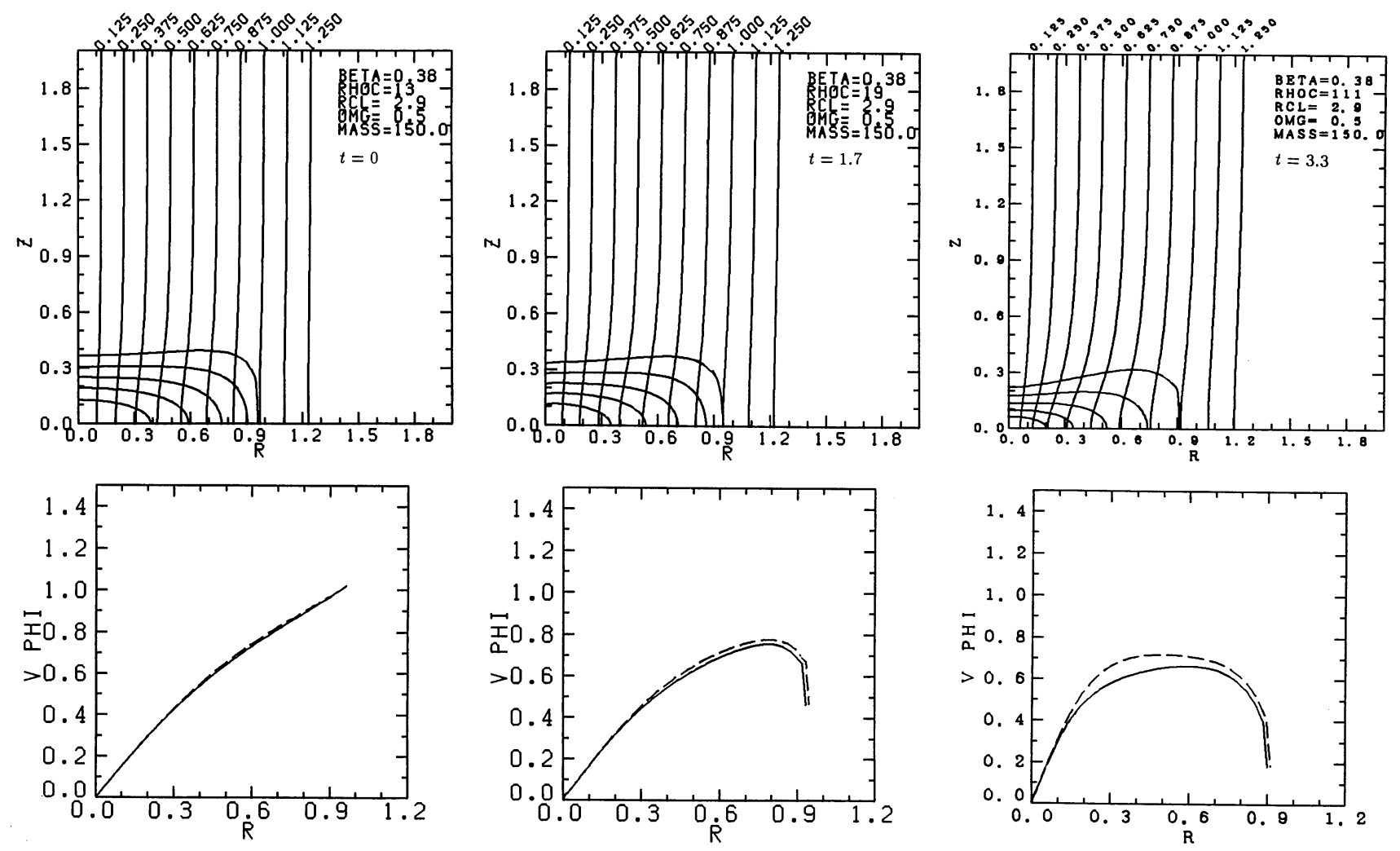

Fig. $6 b$

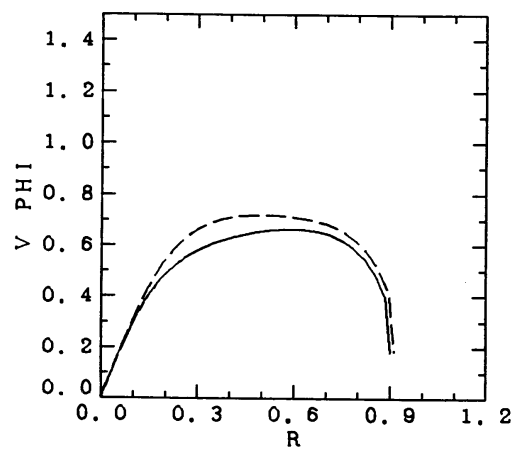

FIG. $6 c$

Fig. 6. - Same as Fig. 1, but for case $\mathrm{C} 2$ at $(a) t=0,(b) t=1.7$, and $(c) t=3.3$. Since the magnetic field is weaker than cases A and B, the maximum density when the cloud departs from the quasi-static evolution is smaller than those of cases $\mathbf{A}$ and $\mathbf{B}$. 

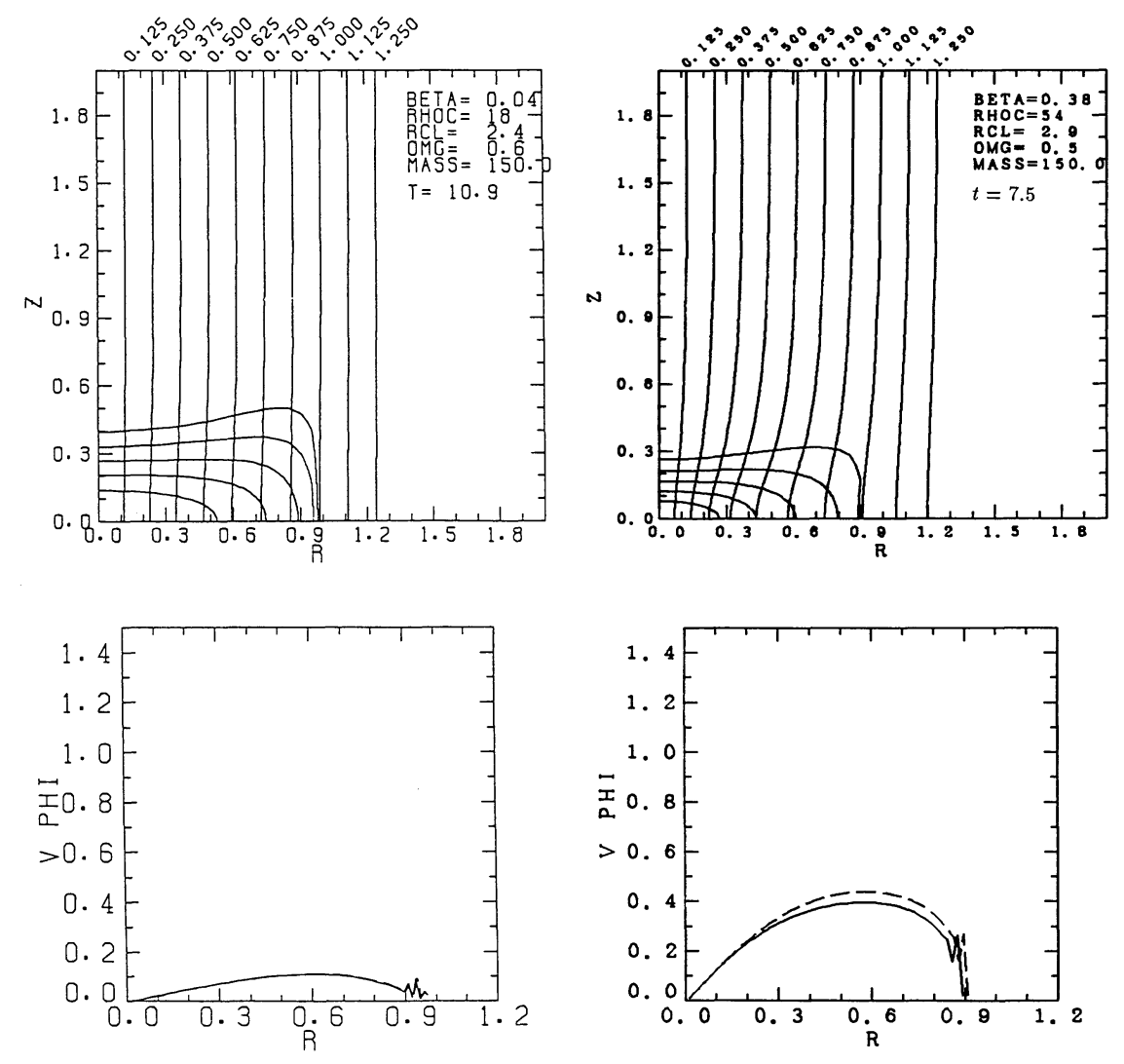

FIG. $7 a$

Fig. $7 b$

Fig. 7. - Same as Fig. 1, but for cases NA2 (a) $t=10.9$ and NC2 $(b) t=7.5$. In these cases, we simulate the evolution of the cloud when the plasma drift is artificially forbidden. The cloud of case NA2 always keeps stable and loses the angular momentum completely. On the other hand, the cloud of case NC2 becomes unstable and enters the dynamically contracting phase ([b] corresponds to the final epoch of the quasi-static evolution), because the angular momentum necessary to support is lost by magnetic braking.

$\beta_{0}=0.0385$ and $\alpha \simeq 0.49$ for $\beta_{0}=0.385$. Case NA2 does not show any significant evolution in this plane. For case NC2, the power index $\alpha \simeq 0.55$ is slightly larger than previous cases. Similar to the result of nonrotating cloud (Nakano 1984), for the rotating cloud the central magnetic field and density correlate well with each other, and the cloud evolves keeping the relation $B_{c} \propto \rho_{c}^{0.4-0.5}$.

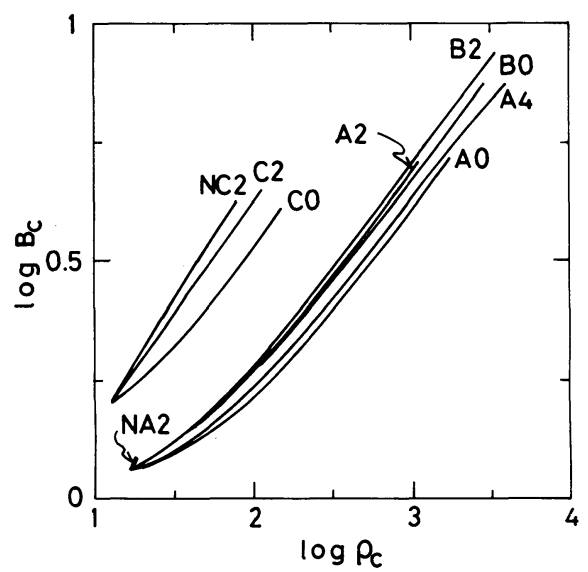

FIG. 8. -The relation between the central density $\rho_{c}$ and the magnetic field strength at the center $B_{c}$

\section{DISCUSSION}

a) Evolution in the $\rho_{c}-G^{1 / 2}\left|d m / d \Phi_{B}\right|_{c}$ Plane

Figure 9 shows the evolutionary paths of cases A0, A2, B0, and $\mathrm{B} 2$ in the plane of the central density, $\rho_{c}$, and the mass-toflux ratio at the center, $G^{1 / 2}\left|d m / d \Phi_{B}\right|_{c}$. The evolutionary direction is shown in arrows. Comparing the cases with and without the magnetic braking, the central density for the case with $\rho_{a}=10^{-2}$ is larger than that for $\rho_{a}=0$. This is because the rotation becomes unable to support the cloud, since the angular momentum is lost by the magnetic braking. Figure 9 also shows that the gradient of each evolutionary path becomes small as the cloud evolves (goes to the right). The mass-to-flux ratio at the center increases by a factor of 2 from the initial value, while the central density increases by a factor of $\gtrsim 100$. The final stage, in which $\rho_{c}$ increases rapidly in Figure 4, corresponds to the stage in which the cloud evolves almost horizontally in Figure 9, that is, $\rho_{c}$ increases greatly, whereas $G^{1 / 2}\left|d m / d \Phi_{B}\right|_{c}$ increases slightly.

The " parent clouds" of cases $\mathrm{A}-\mathrm{C}$ have mass distribution

$$
\frac{d m}{d \Phi}=\left(\frac{N}{2}+1\right) \frac{M_{\mathrm{cl}}}{\Phi_{\mathrm{cl}}}\left(1-\frac{\Phi}{\Phi_{\mathrm{cl}}}\right)^{N / 2},
$$

with $N=1$. In the case of $N=1$ this equation becomes identical with equation (44) of Mouchovias (1976a). In Figure 9, we also plot the $\rho_{c}-G^{1 / 2}\left|d m / d \Phi_{B}\right|_{c}$ relation for the sequence of equilibrium solutions which have a different mass distribution 


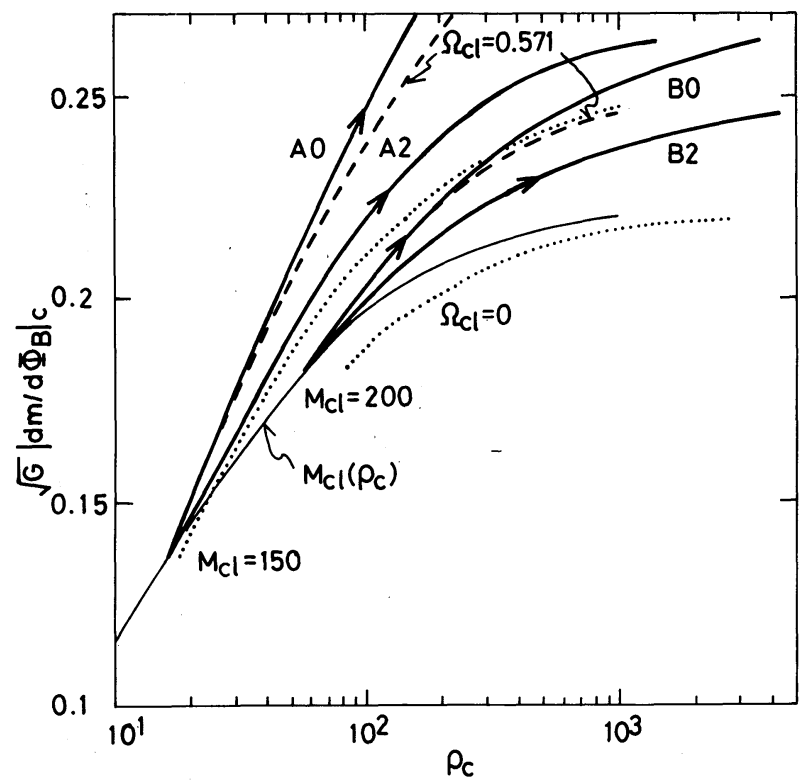

Fig. 9.-The relation between the central density $\rho_{c}$ and the mass-to-flux ratio at the center $G^{1 / 2}\left|d m / d \Phi_{B}\right|_{c}$. Evolutionary paths of cases A0, A2, B0, and $B 2$ are plotted. A series of equilibrium solutions which has distributions of mass and angular momentum like eqs. (4.1) and (4.3) are also illustrated in dashed lines $\left(\Omega_{\mathrm{cl}}=0.571\right)$ and in dotted lines $\left(\Omega_{\mathrm{cl}}=0\right)$. A thin line shows a series of equilibrium solutions which have the same magnetic flux but different masses. This shows that the central density increases by a factor of more than 100 , whereas the mass-to-flux ratio increases only by a factor of 2 .

$m(\Phi)$ from that in $\S \mathrm{II}$, i.e., $N \neq 1$. Since the mass-to-flux ratio at the center is expressed as

$$
G^{1 / 2}\left|\frac{d m}{d \Phi_{B}}\right|_{c}=\left(\frac{N}{2}+1\right) \frac{\beta_{0}^{1 / 2} M_{\mathrm{cl}}}{2^{5 / 2}\left(\pi R_{\mathrm{cl}}\right)^{2}},
$$

by increasing $N$ from unity, we can obtain a series of equilibrium solutions with the same mass and magnetic flux but larger mass-to-flux ratio at the center. Does the equilibrium solution with $N>1$ mimic the cloud in the evolved stage, in which the mass-to-flux ratio at the center increases due to the plasma drift? The angular momentum distribution against mass is taken to be the same as the rigidly rotating uniform spherical cloud. This is reproduced by taking

$$
\begin{aligned}
\frac{d L}{d \Phi}=\left(\frac{N}{2}+1\right) \frac{M_{\mathrm{cl}}}{\Phi_{\mathrm{cl}}} R_{\mathrm{cl}}^{2} \Omega_{\mathrm{cl}} & \left(1-\frac{\Phi}{\Phi_{\mathrm{cl}}}\right)^{N / 2} \\
\times & {\left[1-\left(1-\frac{\Phi}{\Phi_{\mathrm{cl}}}\right)^{(N+2) / 3}\right] . }
\end{aligned}
$$

We show the sequence of these equilibrium solutions with different $N$ and $\Omega_{\mathrm{cl}}=0$ by dotted lines, and the solutions with $\Omega_{\mathrm{cl}}=0.571$ by dashed lines in Figure 9 .

From this figure, it is seen that the evolutionary tracks of the cases with no magnetic braking, $\mathrm{A} 0$ and $\mathrm{B} 0$, almost follow the dashed lines, that is, the sequence of equilibrium clouds with the same angular momentum but various central concentrations of mass and angular momentum. As for cases A2 and B2, since the cloud has a finite angular momentum, the evolutionary paths are different from the equilibrium series without rotation, but it seems that they gradually approach the dotted lines as the angular momentum is lost due to the magnetic braking.

\section{b) Evolutionary Path of Magnetized Clouds}

As for the degree of diminishing magnetic flux at the contraction stage of magnetized clouds, previous works show some controversy. That is, Mouschovias, Paleologou, and Fiedler (1985) asserted that the mass-to-flux ratio increases by several orders of magnitude in the rather low-density cloud $n \lesssim 10^{7-8} \mathrm{~cm}^{-3}$. Contrarily, Nakano $(1982,1984)$ showed that the increase of the mass-to-flux ratio is less than about a factor of 2 , until the ionization degree becomes extremely low due to the high density, say, $n \gtrsim 10^{11} \mathrm{~cm}^{-3}$. Here we will show that the discrepancy can be reconciled by using the $\rho_{c}-G^{1 / 2}\left|d m / d \Phi_{B}\right|_{c}$ relation (Fig. 9) in consideration of the cloud geometry.

Mouschovias, Paleologou, and Fiedler (hereafter MPF; 1985) examined the plane-parallel disk threaded by a parallel magnetic field. The mass-to-flux ratio, $\rho_{c} / B_{c}$, in one of their cases increases by a factor of 170 , while the central density increases from the initial $10^{5} \mathrm{~cm}^{-3}$ to the final $7 \times 10^{6} \mathrm{~cm}^{-3}(\mathrm{a}$ factor of 70; their model $2 r$ ). The evolution proceeds essentially in quasi-static manner. On the other hand, Nakano (1982) considered quasi-static evolution of a flat axisymmetric cloud which collapsed from the spherical cloud penetrated by a uniform magnetic field. In this case, while the central density increases from the initial $4 \times 10^{6} \mathrm{~cm}^{-3}$ to the final $3 \times 10^{8}$ $\mathrm{cm}^{-3}$ (a factor of 80 ), the mass-to-flux ratio at the center $G^{1 / 2}\left|d m / d \Phi_{B}\right|_{c}$ increases only by a factor of 2.1 (his model 4).

As shown in $\S$ II $d$, the time scales of plasma drift for a one-dimensional slab (Shu 1983) and an axisymmetric cloud (Nakano 1984; Mouschovias 1989) give similar values with each other. That is, in the present case, the time scale is expressed as $\tau_{P} \simeq \mathcal{O}(1) \tau_{\mathrm{ff}}^{2} / \tau_{\mathrm{ni}}$ (eq. [2.15]). However, the evolutions are different. From which does the difference come? In next section, we will show that the difference comes from the difference between the evolutionary paths (route) not between the evolutionary time scales (speed).

\section{i) $\rho_{c}$ against Mass-to-Flux Relation for Plane-parallel Disks}

As shown in $\S \mathrm{IV} a$, a series of equilibrium solutions with different mass-to-flux ratios at the center will mimic well the quasi-static evolution of the cloud. In the case of the planeparallel disk threaded by a magnetic field parallel to the disk, taking the $z$-axis perpendicular to the disk and ignoring the inertia term, the hydrostatic equation is written as

$$
\frac{\partial}{\partial z}\left(p+\frac{B^{2}}{8 \pi}\right)=-\rho \frac{\partial \psi}{\partial z}=-2 \pi G \rho \sigma,
$$

with

$$
\sigma \equiv \int_{-z}^{z} \rho d z
$$

where other variables have ordinary meanings. Equation (4.4) is integrated to obtain the relation at the center $(z=0)$ as

$$
p_{c}+\frac{B_{c}^{2}}{8 \pi}-\left(p_{s}+\frac{B_{s}^{2}}{8 \pi}\right)=\frac{\pi G}{2} \sigma_{s}^{2},
$$

where subscripts $c$ and $s$ represent the quantities at the center and at the surface, respectively. Thus, the relation between the nondimensional mass-to-flux ratio $y \equiv\left(c_{s} / \rho_{s}^{1 / 2}\right)(\rho / B)$ and the central density $\rho_{c}^{\prime}=\rho_{c} / \rho_{s}$ is expressed as

$$
\rho_{c}^{\prime}+\frac{\left(\rho_{c}^{\prime}\right)^{2}}{8 \pi y_{c}^{2}}-\frac{1}{8 \pi y_{s}^{2}}=\bar{\rho}_{c}^{\prime},
$$




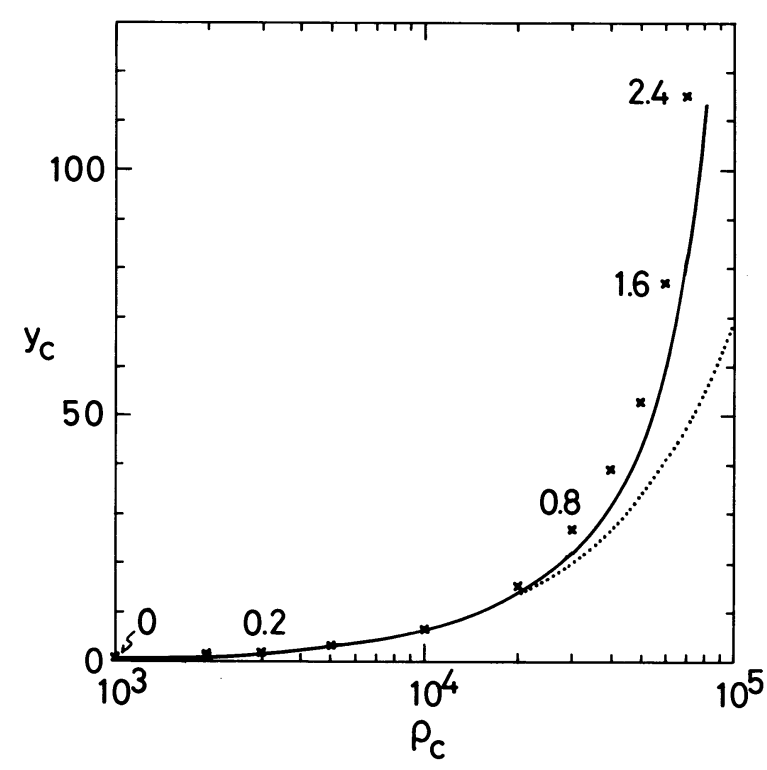

FIG. 10.-The relation between the central density $\rho_{c}$ and the mass-to-flux ratio at the center $y_{c}$ for the plane-parallel layer, which is derived by quasistatic model. The $y$-axis means the one-dimensional mass-to-flux ratio $\left(c_{s} / \rho_{s}^{1 / 2}\right)\left(\rho_{c} / B_{c}\right)$ at the midplane. The parameters are taken same as the dynamical calculation by MPF, and crosses show the result of MPF's model $2 r$. The figure by the cross shows the elapsed time in Myr. This shows that when the central density reaches $\bar{\rho}_{c}^{\prime}$, the mass-to-flux ratio increases much.

where $\bar{\rho}_{c}^{\prime} \equiv \pi G \sigma_{s}^{2} /\left(2 \rho_{s} c_{s}^{2}\right)+1$ represents the central density necessary for the disk to be supported without a magnetic field $(y \rightarrow \infty)$. In Figure 10, we illustrate the $\rho_{c}^{\prime}-y_{c}$ relation (eq. [4.7]) for the cloud with the same parameters taken by MPF as model $2 r$, which corresponds to the case of $\bar{\rho}_{c}^{\prime} \simeq 10^{5}$ and $y_{s} \simeq$ 0.1 . Further, we also show the result of MPF's dynamical calculation by crosses. In contrast to the dotted line which shows the relation of $B_{c}=$ constant, this figure shows that the curve of quasi-static model evolution nicely traces the result of the dynamical calculation by MPF. While the central density is much lower than $\bar{\rho}_{c}^{\prime}$, equation (4.7) indicates that $\rho_{c}^{\prime} \propto y_{c} \propto$ $\rho_{c} / B_{c}$, where we neglected the third term on the left-hand side. This means that in the first phase the density increases while the field strength does not increase, which is also similar to the evolution studied by their dynamical calculation. Equation (4.7) also shows for $\rho_{c}^{\prime} \rightarrow \bar{\rho}_{c}^{\prime}, y_{c} \rightarrow \bar{\rho}_{c}^{\prime} y_{s} \sim 10^{4}$. Because in the self-gravitating cloud $\bar{\rho}_{c}^{\prime}$ must be $\gg 1$, the mass-to-flux ratio $y_{c}$ increases much when the central density approaches $\bar{\rho}_{c}^{\prime}$. As shown in this figure, at the final phase of the evolution of the plane-parallel disk, the evolutionary path points almost vertically upward. This agreement between the static model and dynamical calculation is sufficient to conclude that the evolutionary path shown by the quasi-static model well mimic the true evolution.

ii) Difference of Evolutionary Paths

At the final stage of the quasi-static evolution, the planeparallel disk evolves vertically upward in Figure 10. Contrarily, the flat axisymmetric cloud goes horizontally to the right in Figure 9. That is, the evolutionary path of a one-dimensional slab cloud is different from that of three-dimensional axisymmetric cloud. This difference seems to come from "the saturation of the gravitational field" in the one-dimensional slab cloud, as discussed by Mouschovias (1987) and Nakano (1988). The plane-parallel disk with a magnetic field parallel to the disk studied by MPF is a system in which the magnetic flux decreases with the increase of the central density at the final phase. On the other hand, in the case of the realistic axisymmetric cloud, near the critical state $\left(M_{\mathrm{cl}} \simeq M_{\mathrm{cr}}\right)$ the mass-toflux ratio does not increase easily, although the central density increases. To estimate the increase of the mass-to-flux ratio in the cloud, it is necessary to know the geometry of realistic (flat axisymmetric) clouds. It is shown that the difference comes from that of the evolutionary paths in $\rho_{c}-G^{1 / 2}\left|d m / d \Phi_{B}\right|_{c}$ plane, like the difference between the Henyey and Hayashi tracks in the H-R diagram.

\section{c) Angular Momentum Loss}

If the cloud loses its angular momentum keeping the rigidbody rotation, the time scale of spin-down of the cloud is proportional to $M_{\mathrm{cl}} / R_{\mathrm{cl}}^{2}\left(\beta_{0} / \rho_{a}\right)^{1 / 2}$ (eq. [3.1]). Figure 11 shows the calculated time evolutions of total angular momentum $J$, which decreases in proportion to $\exp \left(-t / \tau_{\|}\right)$. The characteristic time $\tau_{\|}$is estimated as 4.9 (case A2), 43 (case A4), 4.9 (case B2), and 7.6 (case $\mathrm{C} 2$ ). (Case A2 is fitted better with two time constants as $\tau_{\|} \simeq 4.3$ for $t \lesssim 3$ and $\tau_{\|} \simeq 5.2$ for $t \gtrsim 3$. Here we use the above value for convenience.) From this figure, the $e$-folding time of the total angular momentum is approximated as

$$
\tau_{\|} \simeq(0.07-0.1) \frac{M_{\mathrm{cl}}}{R_{\mathrm{cl}}^{2}}\left(\frac{\beta_{0}}{\rho_{a}}\right)^{1 / 2} .
$$

In dimensional form, the time constant becomes

$$
\begin{aligned}
\tau_{\|} \simeq 2.4 \times & 10^{6} \mathrm{yr}\left(\frac{M_{\mathrm{cl}}}{10.7 M_{\odot}}\right)\left(\frac{R_{\mathrm{cl}}}{0.26 \mathrm{pc}}\right)^{-2} \\
& \times\left(\frac{B_{0}}{30 \mu \mathrm{G}}\right)^{-1}\left(\frac{\rho_{a}}{3.8 \times 10^{-23} \mathrm{~g} \mathrm{~cm}^{-3}}\right)^{-1 / 2} .
\end{aligned}
$$

This shows that the simple estimation (3.1) is fairly correct.

\section{SUMMARY}

We have studied the quasi-static evolution of magnetized, rotating, isothermal clouds/cloud cores driving by the plasma drift and magnetic braking.

The time scales of the plasma drift and angular momentum loss are expressed, respectively, as $\tau_{P} \simeq 15.7\left(G \rho_{c}\right)^{-1 / 2} \sim 3$

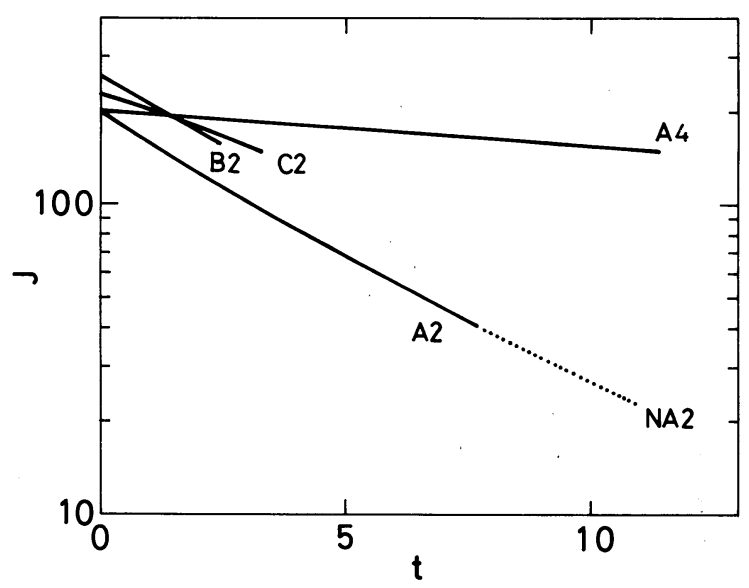

Fig. 11.-The time evolution of angular momentum $J$. This shows clearly that the angular momentum decreases in proportion to $\exp \left(-t / \tau_{\|}\right)$, where $\tau_{\|}$ means a time constant of angular momentum loss. 
$\times 10^{6} \mathrm{yr}\left(\rho_{c} / 10^{5} \mathrm{~cm}^{-3}\right)^{-1 / 2}$ (eq. [2.15]) (Spitzer 1978; Mouschovias 1979; Shu 1983; Nakano 1984; Mouschovias 1989) and $\tau_{\|} \simeq 2.4 \times 10^{6} \mathrm{yr}\left(M_{\mathrm{cl}} / 10.7 M_{\odot}\right)\left(R_{\mathrm{cl}} / 0.26 \mathrm{pc}\right)^{-2}\left(B_{0} / 30\right.$ $\mu \mathrm{G})^{-1}\left(\rho_{a} / 3.8 \times 10^{-23} \mathrm{~g} \mathrm{~cm}^{-3}\right)^{-1 / 2}$ (eq. [4.9]) (Mouschovias and Paleologou 1980). The evolution is determined by these two time scales and available time to evolve to the critical state (or how close the mass of the cloud is to the critical mass).

Cases $A$ and B.-The cloud core is mainly supported by a magnetic field. (a) When the cloud core has the mass much less than the critical mass, the cloud is long lived $\sim(4-7) \times 10^{6} \mathrm{yr}$ and loses almost all the angular momentum $(80 \%)$ in the quasistatic evolution phase. (b) When the cloud core has the mass close to the critical mass, it enters the dynamically contracting phase soon $\left(\sim 10^{6} \mathrm{yr}\right)$, after the plasma drift works effectively in such a cloud core. A fairly large part of the angular momentum $(60 \%)$ remains in the cloud at the final phase of the quasi-static evolution.

Case C.-The cloud (or cloud core) in which rotation also plays an important role. Since the decrease of angular momentum reduces the critical mass, the quasi-static evolution is terminated by the magnetic braking in $\sim 6 \times 10^{6} \mathrm{yr}$. In either case, clouds enter the dynamical contraction phase within several $\times 10^{6} \mathrm{yr}$, after the magnetic flux of the clouds decreases to several times as large as the critical flux.

The density and magnetic field strength at the center of the cloud correlate well with each other as $B_{c} \propto \rho_{c}^{0.4-0.5}$, similar to the nonrotating cloud models with frozen-in magnetic fields (Mouschovias 1976b) and the nonrorating cloud models which evolve driven by the plasma drift (Nakano 1979). From the $\rho_{c}-G^{1 / 2}\left|d m / d \Phi_{B}\right|_{c}$ relation, it is shown that, while the cloud only loses its magnetic flux by a factor of 0.5 , the density near the center increases by a factor more than 100 before the dynamical contraction phase.

The numerical calculations were performed mainly by Hitac S820/80 at Computer Center, University of Tokyo, and partly by Acos S900 at Information Processing Center, Niigata University, and FACOM VP200 at Computer Center, Institute of Space and Aeronautical Sciences. This work was supported in part by Grants-in-Aid for Science Research from the Ministry of Education, Science, and Culture: 01740141 (K. T.), 62540178 (S. I.), and 63611512 (T. N.).

Bonner, W. B. 1956, M.N.R.A.S., 116, 351.
Crutcher, R. M., Troland, T. H., Kazès, I., Heiles, C., Goodman, A. A., and

\section{REFERENCES} Myers, P. C. 1988, preprint.

Ebert, R. 1955, Zs. Ap. 37, 217

Elmegreen, B. G. 1979, Ap. J., 232, 729.

Goldsmith, P. F. 1987, in Interstellar Processes, ed. D. J. Hollenbach and H. A. Thronson (Dordrecht: Reidel), p. 51

Goldsmith, P. F., and Arquilla, R. 1985, in Protostars and Planets II, ed. D. C. Black and M. S. Matthews (Tucson: University Arizona Press), p. 137.

Goodman, A. A., Crutcher, R. M., Heiles, C., Myers, P. C., and Troland, T. H. 1989, Ap. J. (Letters), 338, L61.

Heyer, M. H., Vrba, F. J., Snell, R. L., Schloerb, F. P., Strom, S. E., Goldsmith, P. F., and Strom, K. M. 1987, Ap. J., 321, 855 .

Kiguchi, M., Narita, S., Miyama, S. M., and Hayashi, C. 1987, Ap. J., 317, 830.

Lizano, A. and Shu, F. H. 1989, Ap. J., 342, 824

Mouschovias, T. Ch. $1976 a, A p . J ., 206,753$. 1976b, Ap. J., 207, 141.

1978, in Protostars and Planets, ed. T. Gehrels (Tucson: University of Arizona Press), p. 209

1979, Ap. J., 228, 475.

1987, in Physical Processes in Interstellar Clouds, ed. G. E. Morfill and M. Scholer (Dordrecht: Reidel), p. 491.

1989, in The Physics and Chemistry of Interstellar Molecular Clouds,

Mouschovias, T. Ch., and Paleologou, E. V. 1980, Ap. J., 237, 877.

1986, Ap. J., 308, 781.

Mouschovias, T. Ch., Paleologou, E. V., and Fiedler, R. A. 1985, Ap. J., 291 772 (MPF).

Mouschovias, T. Ch., and Spitzer, L., Jr. 1976, Ap. J., 210, 326.

Nakano, T. 1979, Pub. Astr. Soc. Japan, 31, 697.

1982, Pub. Astr. Soc. Japan, 34, 337

1984, Fund. Cosmic Phys., 9, 139.

1988, in Galactic and Extragalactic Star Formation, ed. R. E. Pudritz and M. Fich (Dordrecht: Reidel), p. 111.

Nakano, T., and Umebayashi, T. 1980, Pub. Astr. Soc. Japan, 32, 613.

Paleologou, E. V., and Mouschovias, T. Ch. 1983, Ap. J., 275, 838.

Priest, E. R. 1982, Solar Magnetohydrodynamics (Dordrecht: Reidel).

Scott, E. H. 1984, Ap. J., 278, 396.

Shu, F. H. 1983, Ap. J., 273, 202.

Spitzer, L., Jr. 1978, Physical Processes in the Interstellar Medium (New York: Wiley).

Tamura, M., et al. 1987, in IAU Symposium 115, Star Forming Regions, ed. M.

Peimbert and J. Jugaku (Dordrecht: Reidel), p. 48.

Tomisaka, K., Ikeuchi, S., and Nakamura, T. 1988a, Ap. J., 326, 208 (Paper I) 1988b, Ap.J., 335, 239 (Paper II). 1989, Ap. J., 341, 220 (Paper III).

ed. G. Winnewisser and J. T. Armstrong (New York: Springer), p. 297

SATORU IkEUCHI: National Astronomical Observatory, Mitaka, Tokyo 181, Japan

TAKASHi NAKAmURA: Research Institute for Fundamental Physics, Kyoto University, Kyoto 606, Japan

Конл TомISAKA: Laboratory of Physics, Faculty of Education, Niigata University, Ikarashi 2-8050, Niigata 950-21, Japan 